\title{
Study of kaon structure using the light-cone quark model
}

\author{
Satvir Kaur and Harleen Dahiya® \\ Dr. B. R. Ambedkar National Institute of Technology, Jalandhar 144011, India
}

(Received 5 August 2019; published 10 October 2019)

\begin{abstract}
We investigate the various distributions explaining the multidimensional structure of kaon at the level of its constituents $(u$ and $\bar{s}$ ) using the light-cone quark model. The overlap form of wave functions associated with the light-cone quark model is adopted for the calculations. The generalized parton distributions (GPDs) of $u$ and $\bar{s}$ quarks are presented for the case when the momentum transfer in the longitudinal direction is nonzero. The dependence of kaon GPDs is studied in terms of the variation of the quark longitudinal momentum fraction, momentum transfer in the longitudinal direction, and total momentum transfer to the final state of the hadron. The transverse impact-parameter dependent GPDs are also studied by taking the Fourier transformation of general GPDs. Further, the quantum phase-space distributions, Wigner distributions, are studied for the case of unpolarized, longitudinally polarized, and transversely polarized partons in an unpolarized kaon. The Wigner distributions are analyzed in the transverse impactparameter plane, the transverse momentum plane, and the mixed plane. Further, to get a complete picture of the kaon in terms of its valence quarks, the variation of longitudinal momentum fraction carried by quark and antiquark in the generalized transverse momentum-dependent parton distributions is studied for different values of transverse quark and antiquark momentum $\left(\mathbf{k}_{\perp}\right)$ as well as for different values of momentum transferred to the kaon in the transverse direction $\left(\boldsymbol{\Delta}_{\perp}\right)$. This has been done for zero as well as nonzero skewedness representing, respectively, the absence and presence of momentum transfer to the final state of the kaon in the longitudinal direction. Furthermore, the possible spin-orbit correlation for $u$ and $\bar{s}$ in the kaon is elaborated in the context of Wigner distributions and generalized transverse momentumdependent parton distributions.
\end{abstract}

DOI: $10.1103 /$ PhysRevD.100.074008

\section{INTRODUCTION}

Quantum chromodynamics (QCD) which is a part of the Standard Model (SM) describes the formation of the hadron by including the strong interrelation between quarks, antiquarks, and gluons. The hadron structure cannot be derived directly from the structure functions and the nonperturbative effects of QCD are the key to understand the complex internal structure of the hadron. This can be attempted through probing where, by knowing the nature of the scattering reaction, one can extract the detailed information about the structure. The probe chosen for the interaction with the hadron is a pointlike particle such as a lepton. The parton distribution functions (PDFs) [1,2] and the form factors (FFs) [3-6] are the basic ingredients to understand the internal hadron structure. The extended information on the internal structure can be explained through the generalized parton distributions (GPDs)

Published by the American Physical Society under the terms of the Creative Commons Attribution 4.0 International license. Further distribution of this work must maintain attribution to the author(s) and the published article's title, journal citation, and DOI. Funded by SCOAP ${ }^{3}$.
[7-11] and the transverse momentum-dependent parton distributions (TMDs) [12,13]: the techniques to understand the three-dimensional picture of the hadron. The GPDs depend on three variables, namely, (a) the quark longitudinal momentum fraction $x$, (b) the momentum transfer in the longitudinal direction $\zeta$, and (c) the total momentum transfer to the final state of the hadron $t=\Delta^{2}$. In general, GPDs are responsible for unraveling the longitudinal and transverse distribution of partons inside the hadron. The Fourier transformation of GPDs provides us with the impact-parameter dependent GPDs carrying the information in terms of the transverse distance from the center of the hadron. The impact-parameter dependent GPDs in the presence of the transverse momentum in the longitudinal direction have been effectively discussed [14]. In the absence of the longitudinal momentum transfer, i.e., $\zeta=0$, the impact-parameter dependent GPDs $\left(x, \mathbf{b}_{\perp}\right)$ end up with a probabilistic interpretation of the parton's density in the fast moving hadron, where $\mathbf{b}_{\perp}$ is the transverse impact-parameter distance [15]. Further, since the GPDs are unable to explain the parton distributions in terms of the transverse momentum carried by the valence parton, the TMDs can be defined as they provide the 
information of parton distributions in terms of the parton's transverse momentum $\left(\mathbf{k}_{\perp}\right)$.

FFs enter into the elastic processes to explain the nonperturbative dynamics of partons in terms of photon virtuality. However, the possibility of the occurrence of inelastic scattering processes increases as compared to the elastic scattering at high photon virtuality. The deep inelastic scattering [16] is helpful in the interpretation of PDFs which explicate the probability of locating the parton carrying the longitudinal momentum fraction $x$ inside the hadron. The GPDs explain through various exclusive processes, the presence of the recoil momentum $\boldsymbol{\Delta}_{\perp}$ as the hadron has different initial and final states. Such processes are named as deeply virtual Compton scattering [17-22] and hard exclusive meson production [23-25]. If the final state has the unpolarized meson with zero spin, one needs to measure the longitudinal cross section [9]. Further, the TMDs which are compatible in explaining the distribution of partons in transverse momentum space are accessible through semi-inclusive deep inelastic scattering and Drell-Yan processes [26-30]. The three-dimensional GPDs are the unification of FFs and PDFs for $\zeta=0$. The $x$ integration of the GPDs leads to the FFs which conceal the location of partons in the transverse direction. Whereas in the forward limit, $\boldsymbol{\Delta}_{\perp}=0$, GPDs produce PDFs.

The quantum phase-space distributions, i.e., Wigner distributions [31,32], are explained as the position and momentum-space distributions of partons in the fast moving hadron. Being limited to the five-dimensional quasiprobabilistic distributions, Wigner distributions interpret in terms of probabilistic quantities, i.e., impact-parameter dependent distributions and TMDs. Furthermore, the Fourier transformation of the Wigner distributions are related to generalized transverse momentum-dependent distributions of partons (GTMDs) [33,34] for $\zeta=0$. Generally, GTMDs are named as mother distributions because GPDs and TMDs can be derived by applying certain integrations and limits. The quark GTMDs in a hadron can be measured through the exclusive double Drell-Yan process where two photons are observed in the final state along with the hadron. The quark GTMDs have been extracted for the case of the nucleon by considering all possible helicities of hadrons and photons [35]. The quark GTMDs can be extracted from the scattering amplitude for the process where the dominating contribution is taken for the transversely polarized photons. The measured quark GTMDs lie in the Efremov-Radyushkin-Brodsky-Lepage (ERBL) region, i.e., $-\zeta<x<\zeta$. The Wigner distributions and GTMDs are also important in calculating the spinorbital angular momentum (spin-OAM) and spin-spin correlations. In addition to this, quark orbital angular momentum is related to the phase-space average of the Wigner distributions.

The probabilistic distributions, i.e., the GPDs and TMDs, have been studied widely in the sense of theories and experiments. The various models which remain successful in explaining the GPDs, both for the nucleon and pion, are the MIT bag model [36], the constituent quark model with a nonrelativistic approach $[37,38]$ as well as with relativistic approach $[39,40]$, the meson cloud model [41], the light-front quark-diquark model [42], and the AdS/QCD inspired light-cone model $[43,44]$ using the Bethe-Salpeter approach $[45,46]$. The GPDs in transverse impact-parameter space have also been studied widely [47-51]. Recently, the unified Wigner distributions have been evaluated extensively using various models by considering different polarization configurations of quarks and gluons in the case of a spin- $\frac{1}{2}$ hadron [52-58]. Further, using these distributions, the spin-spin and spin-OAM correlations have also been studied $[59,60]$. Recently, for the pion, the Wigner distributions have been successfully investigated [61]. Along with the Wigner distributions, the GTMDs have also been studied for the spin $-\frac{1}{2}$ as well as spin-0 hadrons.

In light of the studies done for the case of the pion, we move a step forward to study the internal structure of the kaon in the context of its valence partons by adopting the motivation toward the spin- 0 hadron structure $[33,62]$. It is important to mention here that even though kaon is a spin-0 hadron, it is different from the pion in terms of its constituents that have unequal quark masses (one is light $u$ and other is heavy quark $\bar{s}$ ). Further, the dynamics of valence partons in a spin- 0 meson system are easier to determine as compared to the spin $-\frac{1}{2}$ baryon system as the mesons are composed of a quark-antiquark pair. However, the experiments are more focused on the internal structure of the lowest lying pion and nucleon and there is no experimental data available for the case of the kaon. However, the kaon sea quark distributions can induced by including one charged kaon on an isoscalar target [63]. The kaon-nucleus Drell-Yan process demands several combinations of valence and sea quarks. The combination of differently charged kaons, incident on the isoscalar target, permits one to evaluate valencevalence interactions separately. In other words, the linear combinations of cross sections induced by $\mathrm{K}^{+}$and $\mathrm{K}^{-}$ lead to valence-valence terms while the interaction of $K^{+}$ with the deuteron gives the sea-valence and sea-sea interaction terms.

One of the important models which can be used to investigate the kaon at the level of its constituents is the light-cone QCD inspired model. The light-cone framework $[64,65]$ provides a suitable environment for the description of the hadron's internal structure when it moves relativistically $[65,66]$. The light-cone inspired quark model has been applied to successfully calculate the electromagnetic form factors and compare them with the experimental data [67]. The mesonic light-cone Fock state wave functions are expanded as $|M\rangle=\sum|q \bar{q}\rangle \psi_{q \bar{q}}+\sum|q \bar{q} g\rangle \psi_{q \bar{q} g}+\cdots$, where we choose the minimal Fock state description, i.e., the 
quark-antiquark state, because we study the leading-twist distributions [68].

In this work, we study the various distributions of the $u$ quark as well as the $\bar{s}$ quark in the kaon using the lightcone quark model (LCQM). We have used the overlap representation of the light-cone wave functions. While evaluating the $u$ quark distribution, the other quark, i.e., the $\bar{s}$ quark, is considered as a spectator and vice versa. First of all, we discuss the generalized distributions of quark and antiquark in the kaon for $\zeta \neq 0$. We also discuss the GPDs in transverse position space, i.e., transverse impact-parameter GPDs for the case when the momentum transfer in the longitudinal direction is nonzero $\zeta \neq 0$. We also study the case when the momentum transfer in the longitudinal direction is zero $\zeta=0$. We discuss the Wigner distributions with the quark and antiquark having different polarizations in the kaon. We take the case where the kaon, however, remains unpolarized throughout the calculations. We further explain the kaon GTMDs for the case of $\zeta=0$ as well as $\zeta \neq 0$. In the context of the Wigner distributions and GTMDs, the spinorbit correlation of the $u$ quark and $\bar{s}$ quark in the kaon has also been studied.

The paper is arranged as follows. We provide the detailed description about the general framework of the light-cone quark model and the associated wave functions in Sec. II. In Sec. III, generalized distributions of the quark and antiquark in the kaon are presented for $\zeta \neq 0$ describing the three-dimensional picture of the kaon. Further, the impact-parameter dependent GPDs for nonzero skewedness are evaluated by taking the Fourier transformation from the momentum transferred to the impact-parameter distance in Sec. IV. We present the definitions of Wigner distributions in terms of polarization configurations and the calculations for Wigner distributions, the phase-space distribution describing the five-dimensional picture of the kaon for both $u$ quark and $\bar{s}$ quark in Sec. V. We present the results in the transverse impact-parameter plane, the transverse momentum plane, and in mixed space. In Sec. VI, GTMDs, the mother distributions of valence partons in the kaon, are discussed for $\zeta=0$ as well as $\zeta \neq 0$. The possible spin-orbit correlation for $u$ and $\bar{s}$ quarks in the kaon are calculated, plotted, and discussed in Sec. VII. At the end, we summarize the results in Sec. VIII.

\section{LIGHT-CONE QUARK MODEL}

\section{A. General framework}

If the light-cone momentum fractions and relative momentum coordinates of the hadronic constituents are denoted by $x_{i}=k_{i}^{+} / P^{+}$and $\mathbf{k}_{\perp i}$, then the light-cone Fock state expansion of the hadronic eigenstate, $\left|M\left(P^{+}, \mathbf{P}_{\perp}, S_{z}\right)\right\rangle$ in terms of its constituent eigenstates $|n\rangle$, is defined as $[68,69]$

$$
\begin{aligned}
\left|M\left(P^{+}, \mathbf{P}_{\perp}, S_{z}\right)\right\rangle= & \sum_{n, \lambda_{i}} \int \prod_{i=1}^{n} \frac{\mathrm{d} x_{i} \mathrm{~d}^{2} \mathbf{k}_{\perp i}}{\sqrt{x_{i}} 16 \pi^{3}} 16 \pi^{3} \\
& \times \delta\left(1-\sum_{i=1}^{n} x_{i}\right) \delta^{(2)}\left(\sum_{i=1}^{n} \mathbf{k}_{\perp i}\right) \\
& \times\left|n ; x_{i} P^{+} x_{i} \mathbf{P}_{\perp}+\mathbf{k}_{\perp i}, \lambda_{i}\right\rangle \psi_{n / M}^{\lambda_{i}}\left(x_{i}, \mathbf{k}_{\perp i}\right),
\end{aligned}
$$

where

$$
k_{i}=\left[x_{i} P^{+}, \frac{\left(x_{i} \mathbf{P}_{\perp}+\mathbf{k}_{\perp i}\right)^{2}+m_{i}^{2}}{x_{i} P^{+}}, x_{i} \mathbf{P}_{\perp}+\mathbf{k}_{\perp i}\right] .
$$

Here $m_{i}$ are the masses of $i$ numbers of constituents of the hadron and $\lambda_{i}$ is the helicity of the $i$ th constituent. In Eq. (1), $x_{i} \mathbf{P}_{\perp}+\mathbf{k}_{\perp i}=\mathbf{p}_{\perp i}$ is the physical transverse momenta term and $\psi_{n / M}$ gives the probability amplitudes for finding the on-shell mass constituents of the meson [70]. The $n$-particle Fock states $\left|p_{i}, \mathbf{p}_{\perp i}\right\rangle$ are normalized as follows:

$$
\begin{aligned}
& \left\langle n ; p_{i}^{\prime+}, \mathbf{p}_{\perp i}^{\prime}, \lambda_{i}^{\prime} \mid n ; p_{i}^{+}, \mathbf{p}_{\perp i}, \lambda_{i}\right\rangle \\
& \quad=\prod_{i=1}^{n} 16 \pi^{3} p_{i}^{+} \delta\left(p_{i}^{\prime+}-p_{i}^{+}\right) \delta^{(2)}\left(\mathbf{p}_{\perp i}^{\prime}-\mathbf{p}_{\perp i}\right) \delta_{\lambda_{i}^{\prime} \lambda_{i}} .
\end{aligned}
$$

We use the frame where the general four-vector $A=$ $\left[A^{+}, A^{-}, \mathbf{A}_{\perp}\right]$ components are described as

$A^{ \pm}=A^{0} \pm A^{3}, \quad \mathbf{A}_{\perp}=\left(A^{1}, A^{2}\right) \quad$ and $\quad A^{2}=A^{+} A^{-}-\mathbf{A}_{\perp}^{2}$.

We choose a symmetric light-cone frame for the calculations with $\Delta \rightarrow-\Delta$ symmetry. The initial and final four-momenta of the meson in the symmetric frame are taken as $[68,69]$

$$
\begin{aligned}
& P^{\prime}=\left[(1+\zeta) P^{+}, \frac{M^{2}+\Delta_{\perp}^{2} / 4}{(1+\zeta) P^{+}}, \frac{\Delta_{\perp}}{2}\right], \\
& P^{\prime \prime}=\left[(1-\zeta) P^{+}, \frac{M^{2}+\Delta_{\perp}^{2} / 4}{(1-\zeta) P^{+}},-\frac{\Delta_{\perp}}{2}\right],
\end{aligned}
$$

respectively.

The average four-vector momentum of meson $P^{\mu}=$ $\frac{\left(P^{\prime}+P^{\prime \prime}\right)^{\mu}}{2}$ and the four-vector momentum transfer from the meson $\Delta^{\mu}=P^{\prime \mu}-P^{\prime \prime \mu}$ are given as

$$
\begin{aligned}
& P=\left[P^{+}, \frac{M^{2}+\Delta^{2} / 4}{\left(1-\zeta^{2}\right) P^{+}}, \mathbf{0}_{\perp}\right], \\
& \Delta=\left[2 \zeta P^{+},-\frac{\zeta \Delta_{\perp}^{2}+4 \zeta M^{2}}{2\left(1-\zeta^{2}\right) P^{+}}, \boldsymbol{\Delta}_{\perp}\right],
\end{aligned}
$$


where $\zeta=-\frac{\Delta^{+}}{2 P^{+}}$is the skewedness and $M$ is defined as the meson mass.

\section{B. Light-cone wave functions for the kaon}

The two-particle Fock state expansion in Eq. (1) for the meson $(n=2)$ can be reduced to

$$
\begin{aligned}
|M(P, S)\rangle= & \sum_{\lambda_{1}, \lambda_{2}} \int \frac{d x d^{2} \mathbf{k}_{\perp}}{\sqrt{x(1-x)} 16 \pi^{3}}\left|x, \mathbf{k}_{\perp}, \lambda_{1}, \lambda_{2}\right\rangle \\
& \times \psi_{S_{z}}^{\lambda_{1}, \lambda_{2}}\left(x, \mathbf{k}_{\perp}\right) .
\end{aligned}
$$

Here $\lambda_{1}$ and $\lambda_{2}$ describe the helicities of the quark and antiquark in the meson, respectively.

Since the kaon is a pseudoscalar particle with $S=0$, the light-cone wave functions $\psi_{S_{2}}^{\lambda_{1}, \lambda_{2}}\left(x, \mathbf{k}_{\perp}\right)$ in Eq. (8) can be defined for different combinations of helicities of the quark and spectator antiquark in the kaon as [68]

$$
\begin{aligned}
& \psi_{0}^{\uparrow \uparrow \uparrow}\left(x, \mathbf{k}_{\perp}\right)=-\frac{1}{\sqrt{2}} \frac{k_{1}-i k_{2}}{\sqrt{\mathbf{k}_{\perp}^{2}+l^{2}}} \varphi\left(x, \mathbf{k}_{\perp}\right), \\
& \psi_{0}^{\uparrow, \downarrow}\left(x, \mathbf{k}_{\perp}\right)=\frac{1}{\sqrt{2}} \frac{(1-x) m_{1}+x m_{2}}{\sqrt{\mathbf{k}_{\perp}^{2}+l^{2}}} \varphi\left(x, \mathbf{k}_{\perp}\right), \\
& \psi_{0}^{\downarrow, \uparrow}\left(x, \mathbf{k}_{\perp}\right)=-\frac{1}{\sqrt{2}} \frac{(1-x) m_{1}+x m_{2}}{\sqrt{\mathbf{k}_{\perp}^{2}+l^{2}}} \varphi\left(x, \mathbf{k}_{\perp}\right), \\
& \psi_{0}^{\downarrow, \downarrow}\left(x, \mathbf{k}_{\perp}\right)=-\frac{1}{\sqrt{2}} \frac{k_{1}+i k_{2}}{\sqrt{\mathbf{k}_{\perp}^{2}+l^{2}}} \varphi\left(x, \mathbf{k}_{\perp}\right),
\end{aligned}
$$

with

$$
l^{2}=(1-x) m_{1}^{2}+x m_{2}^{2}-x(1-x)\left(m_{1}-m_{2}\right)^{2} .
$$

The positive (negative) helicity of the quark and the antiquark spectator is denoted by $\uparrow(\downarrow)$. Here, the longitudinal momentum fraction of the quark and the quark transverse momentum are denoted by $x$ and $\mathbf{k}_{\perp}$, respectively. On the other hand, for the antiquark spectator, these terms are described by $(1-x)$ and $-\mathbf{k}_{\perp}$, respectively.
The momentum-space wave function $\varphi\left(x, \mathbf{k}_{\perp}\right)$ in Eq. (9) is described using the Brodsky-Huang-Lepage method [67]. We have

$$
\begin{aligned}
& \varphi\left(x, \mathbf{k}_{\perp}\right) \\
& =A \exp \left[-\frac{\frac{\mathbf{k}_{\perp}^{2}+m_{1}^{2}}{x}+\frac{\mathbf{k}_{\perp}^{2}+m_{2}^{2}}{1-x}}{8 \beta^{2}}-\frac{\left(m_{1}^{2}-m_{2}^{2}\right)^{2}}{8 \beta^{2}\left(\frac{\mathbf{k}_{\perp}^{2}+m_{1}^{2}}{x}+\frac{\mathbf{k}_{\perp}^{2}+m_{2}^{2}}{1-x}\right)}\right],
\end{aligned}
$$

where the parameters $\beta$ and $A$ are defined as the harmonic scale and normalization constant, respectively. Here $m_{1}$ and $m_{2}$ represent the mass of the $u$ quark and $\bar{s}$ quark in the kaon, respectively. The numerical values of the parameters used for the calculations are as follows: $m_{1}=0.25 \mathrm{GeV}, m_{2}=0.5 \mathrm{GeV}$ (with the $u$ quark on shell), $\beta=0.393 \mathrm{GeV}$, and $A=74.2$.

\section{GENERALIZED QUARK AND ANTIQUARK DISTRIBUTIONS FOR THE KAON (GPDS)}

The GPDs are evaluated using the overlap form of wave functions in the LCQM. To carry out with the distributions, the support interval $-1<x<1$ is divided into three regions: (i) the ERBL region $-\zeta<x<\zeta$ where both quark-antiquark pairs are involved, (ii) the Dokshitzer-Gribov-Lipatov-Altarelli-Parisi (DGLAP) region $\zeta<x<1$ for the distributions of the quark, and (iii) the DGLAP region $-1<x<-\zeta$ for the distributions of antiquark. In this work, we have focused on the DGLAP regions for evaluating the distributions of quark and antiquark. In these regions, the conserved number of particle leads to $n \rightarrow n$ overlaps of diagonal elements. For the case of the kaon we have $n=2$ and since it is a spin- 0 particle, the number of GPDs are less as compared to the higher spin particles. The associated GPD for the kaon, defined via the off-diagonal matrix elements of the bilocal field operator, is expressed as [9]

$$
H_{K}(x, \zeta, t)=\left.\frac{1}{2} \int \frac{d z^{-}}{2 \pi} e^{i x P^{+} z^{-}}\left\langle M\left(P^{\prime}\right)\left|\bar{q}\left(-\frac{z}{2}\right) \gamma^{+} q\left(\frac{z}{2}\right)\right| M(P)\right\rangle\right|_{z^{+}=0, \mathbf{z}_{\perp}=\mathbf{0}_{\perp}} .
$$

The overlap form of wave functions for the kaon GPD is achieved by operating the quark field operators on the specific state of the kaon. The $2 \rightarrow 2$ overlaps of wave functions for $H(x, \zeta, t)$ are given as

$$
\begin{aligned}
H_{K}(x, \zeta, t)= & \int \frac{d^{2} \mathbf{k}_{\perp}}{16 \pi^{3}}\left[\psi_{0}^{* \uparrow, \uparrow}\left(x^{\prime \prime}, k^{\prime \prime}\right) \psi_{0}^{\uparrow \uparrow \uparrow}\left(x^{\prime}, k^{\prime}\right)+\psi_{0}^{* \uparrow, \downarrow}\left(x^{\prime \prime}, k^{\prime \prime}\right) \psi_{0}^{\uparrow, \downarrow}\left(x^{\prime}, k^{\prime}\right)\right. \\
& \left.+\psi_{0}^{* \downarrow, \uparrow}\left(x^{\prime \prime}, k^{\prime \prime}\right) \psi_{0}^{\downarrow, \uparrow}\left(x^{\prime}, k^{\prime}\right)+\psi_{0}^{* \downarrow, \downarrow}\left(x^{\prime \prime}, k^{\prime \prime}\right) \psi_{0}^{\downarrow \downarrow \downarrow}\left(x^{\prime}, k^{\prime}\right)\right],
\end{aligned}
$$

where the initial and final momenta $\left(\mathbf{k}_{\perp}^{\prime}\right.$ and $\left.\mathbf{k}_{\perp}^{\prime \prime}\right)$ and longitudinal momentum fractions $\left(x_{1}^{\prime}\right.$ and $\left.x_{1}^{\prime \prime}\right)$ carried by the struck quark in the symmetric frame in the DGLAP domains $\zeta<x<1$ and $-1<x<-\zeta$ are given as 


$$
\mathbf{k}_{\perp}^{\prime}=\mathbf{k}_{\perp 1}+\left(1-x_{1}^{\prime}\right) \frac{\Delta_{\perp}}{2} ; \quad x_{1}^{\prime}=\frac{x_{1}+\zeta}{1+\zeta}
$$

and $\quad \mathbf{k}_{\perp}^{\prime \prime}=\mathbf{k}_{\perp 1}-\left(1-x_{1}^{\prime \prime}\right) \frac{\boldsymbol{\Delta}_{\perp}}{2} ; \quad x_{1}^{\prime \prime}=\frac{x_{1}-\zeta}{1-\zeta}$.

For the spectator, which is an antiquark here, the initial and final states of the momenta and corresponding longitudinal momentum fractions are given as

$$
\mathbf{k}_{\perp s}^{\prime}=\mathbf{k}_{\perp 2}-x_{2}^{\prime} \frac{\boldsymbol{\Delta}_{\perp}}{2} ; \quad x_{2}^{\prime}=\frac{x_{2}}{1+\zeta},
$$

$$
\text { and } \quad \mathbf{k}_{\perp s}^{\prime \prime}=\mathbf{k}_{\perp 2}+x_{2}^{\prime \prime} \frac{\mathbf{\Delta}_{\perp}}{2} ; \quad x_{2}^{\prime \prime}=\frac{x_{2}}{1-\zeta}
$$

It would be important to mention here that for the struck quark, the momentum and longitudinal momentum fraction are taken to be $\mathbf{k}_{\perp 1}=\mathbf{k}_{\perp}$ and $x_{1}=x$, respectively. However, for the antiquark spectator, the respective parameters are $\mathbf{k}_{\perp 2}=-\mathbf{k}_{\perp}$ and $x_{2}=1-x$.

The required conditions that should be satisfied for initial and final states are

$$
\begin{gathered}
\sum_{i=1}^{2} x_{i}^{\prime}=1, \quad \text { and } \quad \sum_{i=1}^{2} x_{i}^{\prime \prime}=1, \\
\sum_{i=1}^{2} \mathbf{k}_{\perp i}^{\prime}=\mathbf{0}_{\perp}, \quad \text { and } \quad \sum_{i=1}^{2} \mathbf{k}_{\perp i}^{\prime \prime}=\mathbf{0}_{\perp} .
\end{gathered}
$$

The antiquark GPDs are defined from quark GPDs as [9]

$$
H^{q}\left(x, \zeta, t, m_{1}, m_{2}\right)=-H^{\bar{q}}\left(-x, \zeta, t, m_{2}, m_{1}\right) .
$$

In the above equation, the masses get reversed in the $\bar{s}$ quark case due to the on-shell mass effect. In other words, when the quark (antiquark) case is taken into account $m_{1}\left(m_{2}\right)$ is considered to be on shell, where $m_{1}\left(m_{2}\right)$ denotes the mass of the $u$ quark ( $\bar{s}$ quark). On the other hand, if we consider the antiquark as an active parton and the quark as the spectator, the conditions described in Eq. (19) should remain the same; however, the antiquark momentum will now be given as $\mathbf{k}_{\perp 1}=-\mathbf{k}_{\perp}$ and the longitudinal momentum fraction by the active antiquark will be given as $x_{1}=-x$. In that case, the quark spectator momentum and longitudinal momentum fraction are taken to be $\mathbf{k}_{\perp 2}=\mathbf{k}_{\perp}$ and $x_{2}=1+x$, respectively.

In the LCQM, the expressions of the GPD $H(x, \zeta, t)$ of the $u$ and $\bar{s}$ quarks for the kaon in the DGLAP regions are expressed as

$$
\begin{aligned}
H^{(u)}= & \int \frac{d^{2} \mathbf{k}_{\perp}}{16 \pi^{3}}\left[\mathbf{k}_{\perp}^{2}-\frac{(1-x)^{2}}{1-\zeta^{2}} \frac{\Delta_{\perp}^{2}}{4}\right. \\
& \left.-\frac{\zeta(1-x)}{1-\zeta^{2}}\left(k_{x} \Delta_{x}+k_{y} \Delta_{y}\right)+\mathcal{M}_{u}^{\prime} \mathcal{M}_{u}^{\prime \prime}\right] \\
& \times \frac{\varphi_{u}^{*}\left(x^{\prime \prime}, \mathbf{k}_{\perp}^{\prime \prime}\right) \varphi_{u}\left(x^{\prime}, \mathbf{k}_{\perp}^{\prime}\right)}{\sqrt{\mathbf{k}_{\perp^{\prime \prime}}^{2}+l_{u}^{\prime \prime 2}} \sqrt{\mathbf{k}_{\perp^{\prime}}^{2}+l_{u}^{\prime 2}}}, \\
H^{(\bar{s})}= & -\int \frac{d^{2} \mathbf{k}_{\perp}}{16 \pi^{3}}\left[\mathbf{k}_{\perp}^{2}-\frac{(1+x)^{2}}{1-\zeta^{2}} \frac{\Delta_{\perp}^{2}}{4}\right. \\
& \left.-\frac{\zeta(1+x)}{1-\zeta^{2}}\left(k_{x} \Delta_{x}+k_{y} \Delta_{y}\right)+\mathcal{M}_{\bar{s}}^{\prime} \mathcal{M}_{\bar{s}}^{\prime \prime}\right] \\
& \times \frac{\varphi_{\bar{s}}^{*}\left(x^{\prime \prime}, \mathbf{k}_{\perp}^{\prime \prime}\right) \varphi_{\bar{s}}\left(x^{\prime}, \mathbf{k}_{\perp}^{\prime}\right)}{\sqrt{\mathbf{k}_{\perp^{\prime \prime}}^{2}+l_{\bar{s}}^{\prime \prime 2}} \sqrt{\mathbf{k}_{\perp^{\prime}}^{2}+l_{\bar{s}}^{\prime 2}}} .
\end{aligned}
$$

Here, we have

$\begin{aligned} \mathcal{M}_{u}^{\prime} & =\frac{1-x}{1+\zeta} m_{1}+\frac{x+\zeta}{1+\zeta} m_{2} \\ \mathcal{M}_{u}^{\prime \prime} & =\frac{1-x}{1-\zeta} m_{1}+\frac{x-\zeta}{1-\zeta} m_{2} \\ \mathcal{M}_{s}^{\prime} & =\frac{1+x}{1+\zeta} m_{2}+\frac{-x+\zeta}{1+\zeta} m_{1} \\ \mathcal{M}_{\bar{s}}^{\prime \prime} & =\frac{1+x}{1-\zeta} m_{2}+\frac{-x-\zeta}{1-\zeta} m_{1} \\ l_{u}^{\prime 2} & =\frac{1-x}{1+\zeta} m_{1}^{2}+\frac{x+\zeta}{1+\zeta} m_{2}^{2}-\frac{(1-x)(x+\zeta)}{(1+\zeta)^{2}}\left(m_{1}-m_{2}\right)^{2},\end{aligned}$

$l_{u}^{\prime \prime 2}=\frac{1-x}{1-\zeta} m_{1}^{2}+\frac{x-\zeta}{1-\zeta} m_{2}^{2}-\frac{(1-x)(x-\zeta)}{(1-\zeta)^{2}}\left(m_{1}-m_{2}\right)^{2}$,

$$
\begin{aligned}
& l_{\bar{s}}^{\prime 2}=\frac{1+x}{1+\zeta} m_{2}^{2}+\frac{-x+\zeta}{1+\zeta} m_{1}^{2}-\frac{(1+x)(\zeta-x)}{(1+\zeta)^{2}}\left(m_{2}-m_{1}\right)^{2} \\
& l^{\prime \prime} \frac{1}{\bar{s}}=\frac{1+x}{1-\zeta} m_{2}^{2}+\frac{-x-\zeta}{1-\zeta} m_{1}^{2}+\frac{(1+x)(x+\zeta)}{(1-\zeta)^{2}}\left(m_{2}-m_{1}\right)^{2}
\end{aligned}
$$

There are primarily four parameters used in the calculations: the mass of the $u$ quark $m_{1}=0.25 \mathrm{GeV}$, the mass of $\bar{s} m_{2}=0.5 \mathrm{GeV}$ (with the $u$ quark being on-shell), the harmonic scale constant $\beta=0.393 \mathrm{GeV}$, and the normalization constant $A=74.2$. Using these parameters, we have calculated the unpolarized GPDs $H(x, \zeta, t)$ of kaon's valence partons corresponding to the $u$ and $\bar{s}$ quarks for 

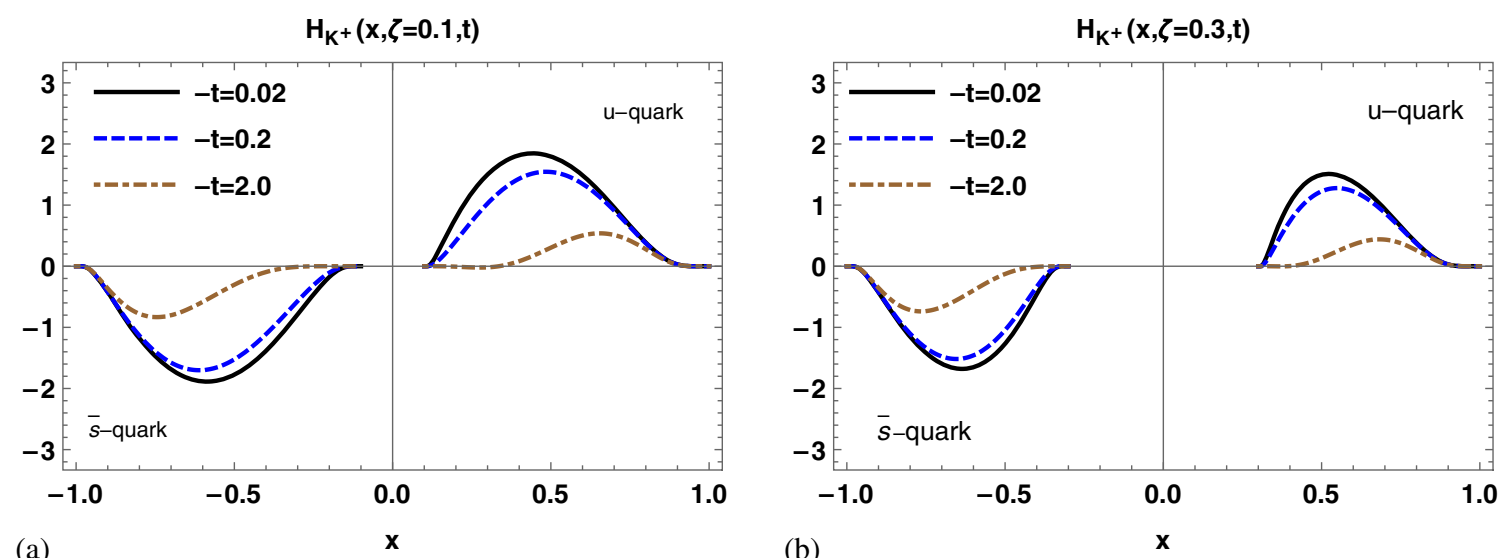

FIG. 1. The variation of the unpolarized kaon GPD $H$ for $u$ and $\bar{s}$ quarks as a function of $x$ at different values of $-t=0.02,0.2$, $2.0 \mathrm{GeV}^{2}$ for skewedness (a) $\zeta=0.1$ and (b) $\zeta=0.3$.

the case when skewedness is nonzero, i.e., $\zeta \neq 0$. First of all, we have fixed the value of $\zeta$ as 0.1 and 0.3 and have shown the variation of the $u$ and $\bar{s}$ GPDs with respect to $x$ for different values of the momentum transfer $-t$ in Figs. 1(a) and 1(b). We observe that the peak of the distribution for the $u$ quark shifts toward higher values of $x$ by when the momentum transfer to the final state $(-t)$ of the kaon increases. The magnitude, however, ceases with the increase in the magnitude of the momentum transfer. In general, the distribution peaks depend on the momentum transfer to the kaon in the longitudinal direction. The higher the longitudinal momentum transfer, the higher the concentration of quark distributions at the higher longitudinal momentum fraction carried by the active quark or antiquark. A similar effect can be seen for the $\bar{s}$ quark case, where the exception lies in the magnitude and polarity. The reason behind the change in magnitude and polarity is the heavier mass of the strange quark. The relation between the flavor decomposition of the kaon is given in Eq. (20) and it is clear that due to the heavy mass, the momentum transfer effect is less in the case of the $\bar{s}$ quark distribution as compared to that in the $u$ quark. It is observed that the distribution is maximum when the quark longitudinal momentum fraction and total momentum transfer are lower. Further, it can be seen that, at $x \rightarrow 1$, the distribution does not depend on the values of $t$. This is because at $x \rightarrow 1$, the total longitudinal momentum fraction is carried by the struck quark, which was first distributed into all the valence partons in the kaon which makes the contribution from the partons (except the active parton) negligible. The same happens with the active antiquark in the limit $x \rightarrow-1$.

In Fig. 2, we have fixed the momentum transfer $-t=$ $0.5 \mathrm{GeV}^{2}$ and presented the GPDs with respect to $x$ for different values of $\zeta$. We observe that at a lower value of $\zeta$, the distribution shows a maximum peak with respect to $x$, whereas the magnitude of the peak decreases as the value of $\zeta$ increases. The peak also moves toward higher $|x|$ with the increasing values of $\zeta$. This implies that the longitudinal momentum fraction carried by the quark depends on the momentum transfer in the longitudinal direction. As the longitudinal momentum transfer increases, the concentration of quarks moves toward higher values of the longitudinal momentum fraction; however, the overall magnitude of the peak decreases.

To get a deeper understanding of the relation between the parameters, we present the quark and antiquark distributions of the kaon by fixing the value of $x$ and observing it as a function of $-t$ and $\zeta$ in Figs. 3(a) and 3(b). Here, it can be seen that at $\zeta=0$, the distribution is different for the different total momentum transferred $-t$ to the kaon from the initial to final state. We also observe that in the $\bar{s}(u)$ quark case, the distribution smoothly rises (falls) when $\zeta$ increases.

From the discussions of Figs. 1-3, it can be concluded that the distribution $H(x, \zeta, t)$ is zero for $x=\zeta$ and $x=-\zeta$ for the $u$ and $\bar{s}$ quarks, respectively. The absence of the distribution in this limit is the supportive domain of the

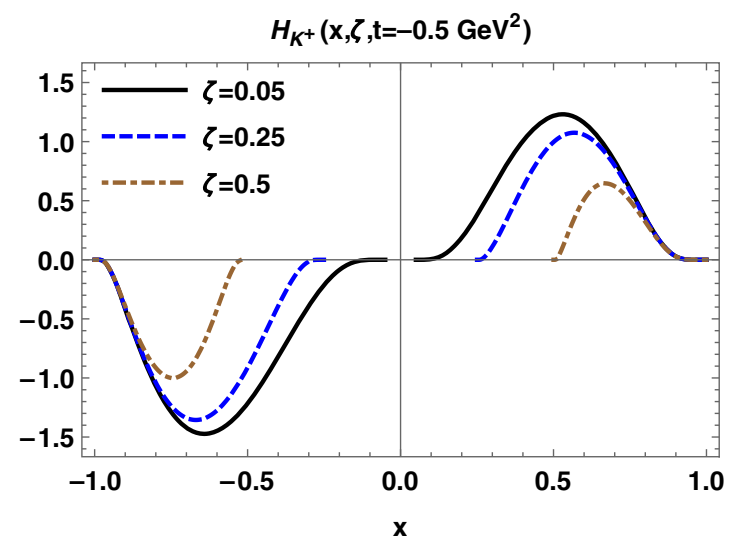

FIG. 2. The variation of the unpolarized kaon GPD $H$ for $u$ and $\bar{s}$ quarks as a function of $x$ at constant $-t=0.5 \mathrm{GeV}^{2}$ and different values of $\zeta=0.05,0.25,0.5$. 

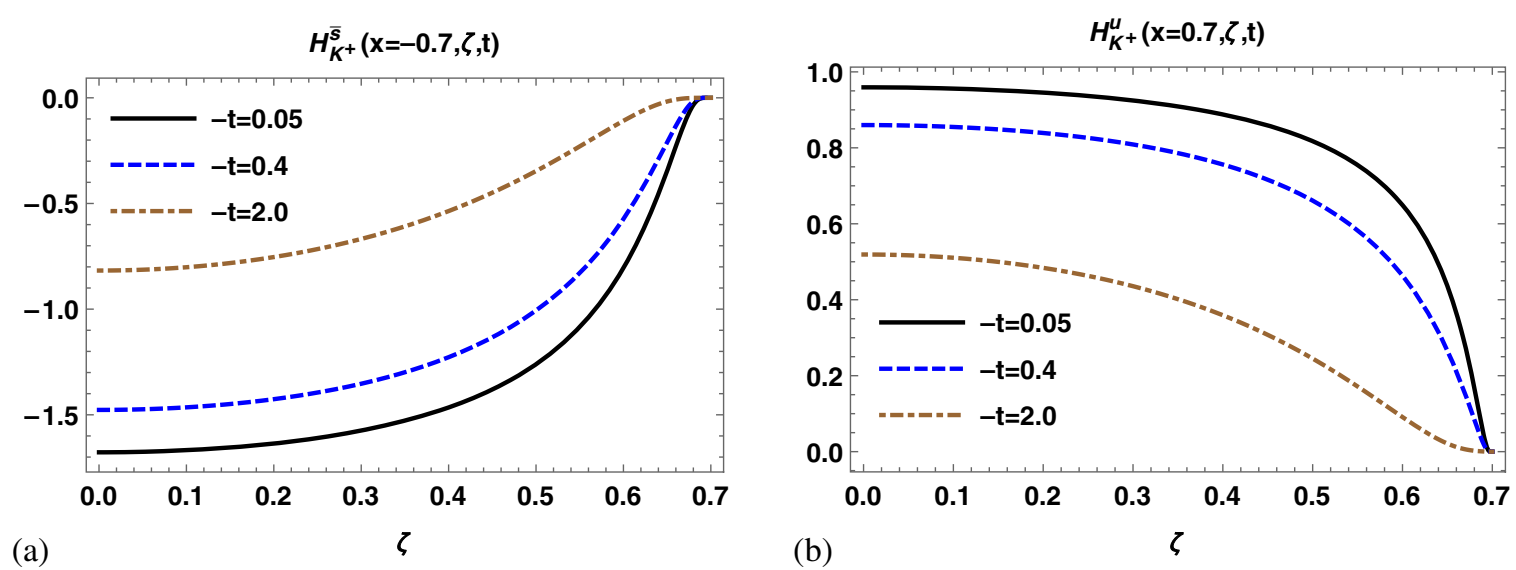

FIG. 3. The variation of the unpolarized kaon GPD $H$ with $\zeta$ at different values of $-t=0.05,0.4,2.0 \mathrm{GeV}^{2}$ for (a) $-x=0.7$ and (b) $x=0.7$ corresponding to $\bar{s}$ and $u$ quark, respectively.

model for evaluating the valence quark (antiquark) distributions which is possible at only lower values of $x$. At higher values of $x$, the sea quarks dominate and experiments are being planned to extend the measurements in this $x$ region. Even though the evaluation of the total distributions includes the collective effects of valence and sea quarks, we are ignoring the contribution coming from the sea quarks in the present work.

\section{TRANSVERSE IMPACT-PARAMETER DEPENDENT GPDS}

We have now taken the two-dimensional Fourier transformation of the GPD with respect to the transverse momentum transfer $\left(\boldsymbol{\Delta}_{\perp}\right)$, i.e., $\boldsymbol{\Delta}_{\perp} \rightarrow \mathbf{b}_{\perp}$. The transverse impact-parameter dependent quark GPD of the kaon in this case can be expressed as [14]

$$
\mathcal{H}^{q}(x, \zeta, b)=\frac{1}{(2 \pi)^{2}} \int d^{2} \mathbf{D}_{\perp} e^{-i \mathbf{D}_{\perp} \cdot \mathbf{b}_{\perp}} H^{q}(x, \zeta, t) .
$$

Here $\mathbf{b}_{\perp}$ describes the impact-parameter position in the transverse direction, which comes after applying the Fourier transformation of the GPD with respect to $\mathbf{D}_{\perp}$. The variable $\mathbf{D}_{\perp}$ is because of the nonzero skewedness and is related to $\boldsymbol{\Delta}_{\perp}$ as

$$
\mathbf{D}_{\perp}=\frac{\mathbf{P}_{\perp}^{\prime \prime}}{1-\zeta}-\frac{\mathbf{P}_{\perp}^{\prime}}{1+\zeta}=\frac{\Delta_{\perp}}{1-\zeta^{2}}
$$

The valence partons of the kaon located at the transverse impact-parameter position $\mathbf{b}_{\perp}$ are probed by assuming the kaon's initial and final states to be positioned at a fixed point but the relative distance between them is changed by the amount $\zeta \mathbf{b}_{\perp}$. In the absence of longitudinal momentum transferred to the kaon, i.e., for $\zeta=0$, the Fourier transform of the GPD $\left(x, 0,-\Delta^{2}\right)$ provides the parton distribution as a function of $x$ and the transverse position $\mathbf{b}_{\perp}$ [15]. We have

$$
q\left(x, \mathbf{b}_{\perp}\right)=\int \frac{d^{2} \boldsymbol{\Delta}_{\perp}}{(2 \pi)^{2}} e^{-i \mathbf{b}_{\perp} . \boldsymbol{\Delta}_{\perp}} H^{q}\left(x, 0,-\boldsymbol{\Delta}_{\perp}^{2}\right) .
$$

In Figs. 4(a) and 4(b), we fix the skewedness $\zeta=0.2$ and present the three-dimensional picture of the transverse impact-parameter dependent GPD $\mathcal{H}(x, \zeta, b)$ with respect to the longitudinal momentum fraction carried by the quark (antiquark) $x(-x)$ and the distance from the transverse center of momentum for the $\bar{s}$ and $u$ quarks. If we let ourselves observe $\mathcal{H}$ with respect to only $b$, we find that the distribution is maximum at the center for both quarks. In the context of $x$ we observe that, at the point when there is no transverse distance from the center of momentum of the kaon, the distribution peaks have dependence on $x$. The amplitude of the peak changes with the changing values of $x$. While there is no distribution of valence partons in the kaon at the higher values of $b$, which implies that if we keep on increasing the transverse distance, the distribution becomes negligible. When we compare the distributions of $\bar{s}$ and $u$ quarks, it can be clearly seen that the magnitude of the distribution lies at slightly higher values of $|x|$ for the case of $\bar{s}$ as compared to that in the case of $u$. On the other side, if one concentrates on the variation of $\mathcal{H}$ with respect to $x$, one can see that with the increase in transverse position $b$, the distribution peak shifts toward the lower values of $|x|$.

Furthermore, we present the distribution $\mathcal{H}(x, \zeta, b)$ as a function of $\zeta$ and $\mathbf{b}_{\perp}$ in Figs. 5(a) and 5(b) by fixing the value of $x$ at -0.7 and 0.7 for the $\bar{s}$ and $u$ quarks, respectively. We notice that the distribution peaks become wider and the amplitude decreases at the higher values of $\zeta$ for the $u$ quark and $\bar{s}$ quark. The spread is maximum, when the momentum transfer in the longitudinal direction is minimum. It implies that if no longitudinal momentum transfer is there, the same longitudinal momentum fraction is carried by the active parton in the initial as well as in the final state. This leads to the maximum spread for $\zeta=0$. 

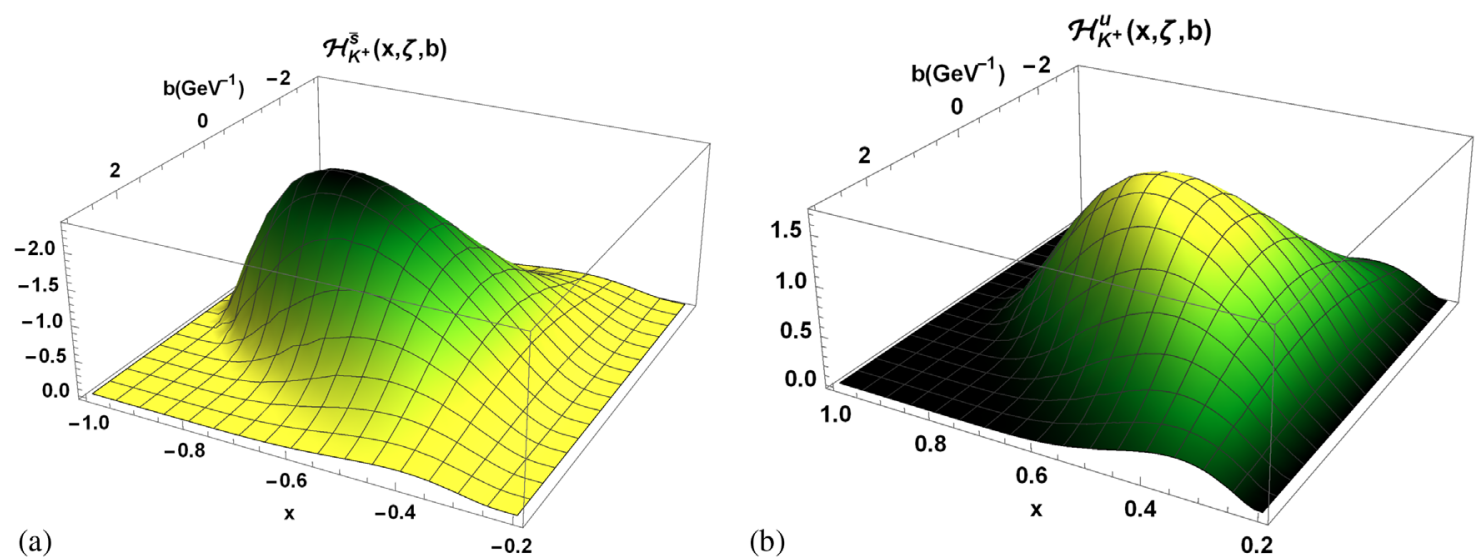

FIG. 4. The 3D plots showing the variation of the transverse impact-parameter dependent parton distribution $\mathcal{H}$ of the (a) $\bar{s}$ quark and (b) $u$ quark, with respect to $x$ and $b$ for a fixed value of $\zeta=0.2$.
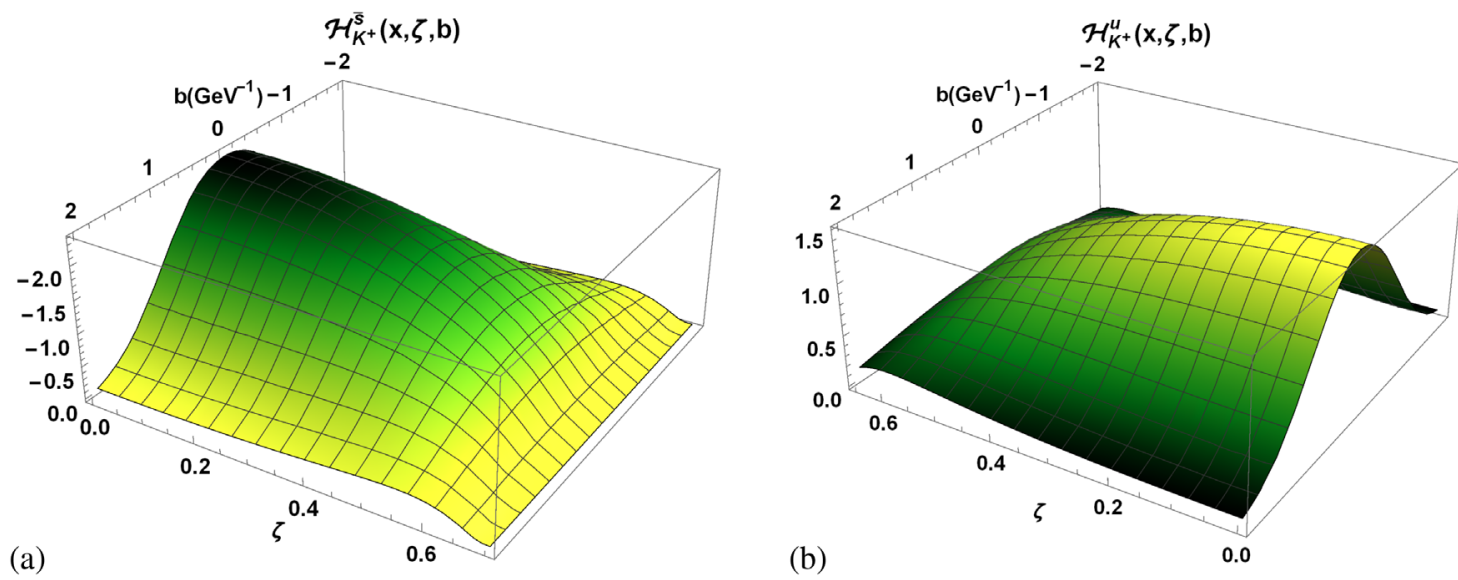

FIG. 5. The 3D plots showing the variation of the transverse impact-parameter dependent parton distribution $\mathcal{H}$ with respect to $\zeta$ and $b$ at (a) $-x=0.7$ for the $\bar{s}$ quark and (b) at $x-0.7$ for the $u$ quark.

\section{WIGNER DISTRIBUTIONS OF THE $u$ QUARK AND $\bar{s}$ QUARK IN THE UNPOLARIZED KAON}

The quantum phase-space distributions also known as Wigner distributions describe the five-dimensional picture of a hadron. Specifically, it defines three momentum and two position coordinates [52,53] and is defined as

$\rho^{[\Gamma]}\left(\mathbf{b}_{\perp}, \mathbf{k}_{\perp}, x, S\right) \equiv \int \frac{d^{2} \boldsymbol{\Delta}_{\perp}}{(2 \pi)^{2}} e^{-i \boldsymbol{\Delta}_{\perp} \cdot \mathbf{b}_{\perp}} \hat{W}^{[\Gamma]}\left(\boldsymbol{\Delta}_{\perp}, \mathbf{k}_{\perp}, x, S\right)$.

In the Drell-Yan frame $\left(\Delta^{+}=0\right)$, the Wigner operator or correlator $\hat{W}^{[\Gamma]}\left(\boldsymbol{\Delta}_{\perp}, \mathbf{k}_{\perp}, x, S\right)$ at fixed light-cone time $z^{+}=0$ is defined by

$$
\begin{aligned}
& \hat{W}^{[\Gamma]}\left(\Delta_{\perp}, \mathbf{k}_{\perp}, x ; S\right)=\frac{1}{2} \int \frac{d z^{-} d^{2} z_{\perp}}{(2 \pi)^{3}} e^{i k \cdot z} \\
& \quad \times\left.\left\langle M\left(P^{\prime \prime} ; S\right)\left|\bar{\psi}\left(-\frac{z}{2}\right) \Gamma \mathcal{W}_{\left[-\frac{z}{2}, \frac{z}{2}\right]} \psi\left(\frac{z}{2}\right)\right| M\left(P^{\prime} ; S\right)\right\rangle\right|_{z^{+}=0},
\end{aligned}
$$

where $\Gamma$ denotes the twist-2 Dirac $\gamma$-matrices $\gamma^{+}, \gamma^{+} \gamma_{5}$, $i \sigma^{j+} \gamma_{5}(j=1$ or 2 , depending on the polarization direction of quark) corresponding to the unpolarized, longitudinally polarized, and transversely polarized partons. The initial (final) momentum state and spin of hadron is described by $P^{\prime}\left(P^{\prime \prime}\right)$ and $S$, respectively. The symbol $\mathcal{W}_{\left[-\frac{z}{2}, \frac{z}{2}\right]}$ describes the Wilson line which ensures the SU(3) color gauge invariance of the operator $\hat{W}$. As we have restricted ourselves to the calculations of the kaon distributions in the present work, the kaon being a pseudoscalar meson will remain unpolarized throughout the calculations $(S=0)$. Therefore, spin will not be included in Eqs. (32) and (33).

By combining the different polarization configurations of the quark within the unpolarized hadron (having spin-0), the Wigner distributions are defined as follows [61]. For the unpolarized quark in the unpolarized hadron, we have

$$
\rho_{U U}\left(\mathbf{b}_{\perp}, \mathbf{k}_{\perp}, x\right)=\rho^{\left[\gamma^{+}\right]}\left(\mathbf{b}_{\perp}, \mathbf{k}_{\perp}, x\right),
$$


for the longitudinally polarized quark in the unpolarized hadron, we have

$$
\rho_{U L}\left(\mathbf{b}_{\perp}, \mathbf{k}_{\perp}, x\right)=\rho^{\left[\gamma^{+} \gamma_{5}\right]}\left(\mathbf{b}_{\perp}, \mathbf{k}_{\perp}, x\right),
$$

and for the transversely polarized quark in the unpolarized hadron, we have

$$
\rho_{U T}^{j}\left(\mathbf{b}_{\perp}, \mathbf{k}_{\perp}, x\right)=\rho^{\left[i \sigma^{j+} \gamma_{5}\right]}\left(\mathbf{b}_{\perp}, \mathbf{k}_{\perp}, x\right) .
$$

By using Eq. (1) in Eq. (33), we get the Wigner correlation operator $\hat{W}^{[\Gamma]}\left(\boldsymbol{\Delta}_{\perp}, \mathbf{k}_{\perp}, x\right)$ for $\Gamma=\gamma^{+}, \gamma^{+} \gamma_{5}, i \sigma^{j+} \gamma_{5}$ in the overlap form as

$$
\begin{aligned}
\hat{W}^{\left[\gamma^{+}\right]}\left(\mathbf{\Delta}_{\perp}, \mathbf{k}_{\perp}, x\right)= & \frac{1}{16 \pi^{3}}\left[\psi_{0}^{* \uparrow, \uparrow}\left(x, \mathbf{k}_{\perp}^{\prime \prime}\right) \psi_{0}^{\uparrow \uparrow \uparrow}\left(x, \mathbf{k}_{\perp}^{\prime}\right)+\psi_{0}^{* \downarrow, \uparrow}\left(x, \mathbf{k}_{\perp}^{\prime \prime}\right) \psi_{0}^{\downarrow, \uparrow}\left(x, \mathbf{k}_{\perp}^{\prime}\right)+\psi_{0}^{* \uparrow, \downarrow}\left(x, \mathbf{k}_{\perp}^{\prime \prime}\right) \psi_{0}^{\uparrow, \downarrow}\left(x, \mathbf{k}_{\perp}^{\prime}\right)\right. \\
& \left.+\psi_{0}^{* \downarrow, \downarrow}\left(x, \mathbf{k}_{\perp}^{\prime \prime}\right) \psi_{0}^{\downarrow, \downarrow}\left(x, \mathbf{k}_{\perp}^{\prime}\right)\right],
\end{aligned}
$$

$$
\begin{aligned}
\hat{W}^{\left[\gamma^{+} \gamma_{5}\right]}\left(\boldsymbol{\Delta}_{\perp}, \mathbf{k}_{\perp}, x\right)= & \frac{1}{16 \pi^{3}}\left[\psi_{0}^{* \uparrow, \uparrow}\left(x, \mathbf{k}_{\perp}^{\prime \prime}\right) \psi_{0}^{\uparrow \uparrow \uparrow}\left(x, \mathbf{k}_{\perp}^{\prime}\right)-\psi_{0}^{* \downarrow, \uparrow}\left(x, \mathbf{k}_{\perp}^{\prime \prime}\right) \psi_{0}^{\downarrow, \uparrow}\left(x, \mathbf{k}_{\perp}^{\prime}\right)+\psi_{0}^{* \uparrow, \downarrow}\left(x, \mathbf{k}_{\perp}^{\prime \prime}\right) \psi_{0}^{\uparrow, \downarrow}\left(x, \mathbf{k}_{\perp}^{\prime}\right)\right. \\
& \left.-\psi_{0}^{* \downarrow, \downarrow}\left(x, \mathbf{k}_{\perp}^{\prime \prime}\right) \psi_{0}^{v}\left(x, \mathbf{k}_{\perp}^{\prime}\right)\right],
\end{aligned}
$$

$$
\begin{aligned}
\hat{W}^{\left[i \sigma^{j+} \gamma_{5}\right]}\left(\boldsymbol{\Delta}_{\perp}, \mathbf{k}_{\perp}, x\right)= & \frac{1}{16 \pi^{3}} \epsilon_{\perp}^{i j}\left[(-i)^{i} \psi_{0}^{* \uparrow, \uparrow}\left(x, \mathbf{k}_{\perp}^{\prime \prime}\right) \psi_{0}^{\downarrow, \uparrow}\left(x, \mathbf{k}_{\perp}^{\prime}\right)+(i)^{i} \psi_{0}^{* \downarrow, \uparrow}\left(x, \mathbf{k}_{\perp}^{\prime \prime}\right) \psi_{0}^{\uparrow, \uparrow}\left(x, \mathbf{k}_{\perp}^{\prime}\right)\right. \\
& \left.+(-i)^{i} \psi_{0}^{* \uparrow, \downarrow}\left(x, \mathbf{k}_{\perp}^{\prime \prime}\right) \psi_{0}^{\downarrow, \downarrow}\left(x, \mathbf{k}_{\perp}^{\prime}\right)+(i)^{i} \psi_{0}^{* \downarrow, \downarrow}\left(x, \mathbf{k}_{\perp}^{\prime \prime}\right) \psi_{0}^{\uparrow, \downarrow}\left(x, \mathbf{k}_{\perp}^{\prime}\right)\right] .
\end{aligned}
$$

The explicit expressions for quark Wigner distributions in the kaon using Eqs. (32), (37), (38) and (39) are given as

$$
\begin{aligned}
& \rho_{U U}^{u}\left(\mathbf{b}_{\perp}, \mathbf{k}_{\perp}, x\right)= \frac{1}{16 \pi^{3}} \int \frac{d \Delta_{x} d \Delta_{y}}{(2 \pi)^{2}} \cos \left(\Delta_{x} b_{x}+\Delta_{y} b_{y}\right)\left[\mathbf{k}_{\perp}^{2}-(1-x)^{2} \frac{\Delta_{\perp}^{2}}{4}+\left((1-x) m_{1}+x m_{2}\right)^{2}\right] \\
& \times \frac{\varphi_{u}^{\dagger}\left(x, \mathbf{k}_{\perp}^{\prime \prime}\right) \varphi_{u}\left(x, \mathbf{k}_{\perp}^{\prime}\right)}{\sqrt{\mathbf{k}_{\perp^{\prime \prime}}^{2}+l_{u}^{2}} \sqrt{\mathbf{k}_{\perp^{\prime}}^{2}+l_{u}^{2}}}, \\
& \rho_{U L}^{u}\left(\mathbf{b}_{\perp}, \mathbf{k}_{\perp}, x\right)= \frac{1}{16 \pi^{3}} \int \frac{d \Delta_{x} d \Delta_{y}}{(2 \pi)^{2}} \sin \left(\Delta_{x} b_{x}+\Delta_{y} b_{y}\right)(1-x)\left(k_{y} \Delta_{x}-k_{x} \Delta_{y}\right) \frac{\varphi_{u}^{\dagger}\left(x, \mathbf{k}_{\perp}^{\prime \prime}\right) \varphi_{u}\left(x, \mathbf{k}_{\perp}^{\prime}\right)}{\sqrt{\mathbf{k}_{\perp^{\prime \prime}}^{2}+l_{u}^{2}} \sqrt{\mathbf{k}_{\perp^{\prime}}^{2}+l_{u}^{2}}}, \\
& \rho_{U T}^{u}\left(\mathbf{b}_{\perp}, \mathbf{k}_{\perp}, x\right)=-\frac{1}{16 \pi^{3}} \int \frac{d \Delta_{x} d \Delta_{y}}{(2 \pi)^{2}} \sin \left(\Delta_{x} b_{x}+\Delta_{y} b_{y}\right)\left((1-x) m_{1}+x m_{2}\right)(1-x) \Delta_{y} \frac{\varphi_{u}^{\dagger}\left(x, \mathbf{k}_{\perp}^{\prime \prime}\right) \varphi_{u}\left(x, \mathbf{k}_{\perp}^{\prime}\right)}{\sqrt{\mathbf{k}_{\perp \prime}^{2}+l_{u}^{2}} \sqrt{\mathbf{k}_{\perp^{\prime}}^{2}+l_{u}^{2}}} .
\end{aligned}
$$

The flavor decompositions of valence partons in the kaon are associated with each other through the relation

$$
\rho^{u}\left(\mathbf{b}_{\perp}, \mathbf{k}_{\perp}, x, m_{1}, m_{2}\right)=-\rho^{\bar{s}}\left(\mathbf{b}_{\perp},-\mathbf{k}_{\perp},-x, m_{2}, m_{1}\right) .
$$

The explicit expressions for Wigner distributions of the antiquark after implementing Eq. (43) and the conditions, we get

$$
\begin{aligned}
\rho_{U U}^{\bar{s}}\left(\mathbf{b}_{\perp}, \mathbf{k}_{\perp}, x\right)= & -\frac{1}{16 \pi^{3}} \int \frac{d \Delta_{x} d \Delta_{y}}{(2 \pi)^{2}} \cos \left(\Delta_{x} b_{x}+\Delta_{y} b_{y}\right)\left[\mathbf{k}_{\perp}^{2}-(1+x)^{2} \frac{\Delta_{\perp}^{2}}{4}+\left((1+x) m_{2}-x m_{1}\right)^{2}\right] \\
& \times \frac{\varphi_{\bar{s}}^{\dagger}\left(x, \mathbf{k}_{\perp}^{\prime \prime}\right) \varphi_{\bar{s}}\left(x, \mathbf{k}_{\perp}^{\prime}\right)}{\sqrt{\mathbf{k}_{\perp^{\prime \prime}}^{2}+l_{\bar{s}}^{2}} \sqrt{\mathbf{k}_{\perp^{\prime}}^{2}+l_{\bar{s}}^{2}}},
\end{aligned}
$$




$$
\begin{gathered}
\rho_{U L}^{\bar{s}}\left(\mathbf{b}_{\perp}, \mathbf{k}_{\perp}, x\right)=\frac{1}{16 \pi^{3}} \int \frac{d \Delta_{x} d \Delta_{y}}{(2 \pi)^{2}} \sin \left(\Delta_{x} b_{x}+\Delta_{y} b_{y}\right)(1+x)\left(k_{x} \Delta_{y}-k_{y} \Delta_{x}\right) \frac{\varphi_{\bar{s}}^{\dagger}\left(x, \mathbf{k}_{\perp}^{\prime \prime}\right) \varphi_{\bar{s}}\left(x, \mathbf{k}_{\perp}^{\prime}\right)}{\sqrt{\mathbf{k}_{\perp^{\prime \prime}}^{2}+l_{\bar{s}}^{2}} \sqrt{\mathbf{k}_{\perp^{\prime}}^{2}+l_{\bar{s}}^{2}}}, \\
\rho_{U T}^{\bar{s}}\left(\mathbf{b}_{\perp}, \mathbf{k}_{\perp}, x\right)=\frac{1}{16 \pi^{3}} \int \frac{d \Delta_{x} d \Delta_{y}}{(2 \pi)^{2}} \sin \left(\Delta_{x} b_{x}+\Delta_{y} b_{y}\right)\left((1+x) m_{2}-x m_{1}\right)(1+x) \Delta_{y} \frac{\varphi_{\bar{s}}^{\dagger}\left(x, \mathbf{k}_{\perp}^{\prime \prime}\right) \varphi_{\bar{s}}\left(x, \mathbf{k}_{\perp}^{\prime}\right)}{\sqrt{\mathbf{k}_{\perp^{\prime \prime}}^{2}+l_{\bar{s}}^{2}} \sqrt{\mathbf{k}_{\perp^{\prime}}^{2}+l_{\bar{s}}^{2}}} .
\end{gathered}
$$

Since the Wigner distributions are interpreted with $\zeta=0$, the initial and final state momenta and longitudinal momentum fractions can be used by putting $\zeta=0$ in Eqs. (14)-(19).

Further, we can obtain the purely transverse Wigner distributions by integrating them over $x$ as

$$
\rho_{U X}\left(\mathbf{b}_{\perp}, \mathbf{k}_{\perp}\right) \equiv \int d x \rho_{U X}\left(\mathbf{b}_{\perp}, \mathbf{k}_{\perp}, x\right),
$$

where $X$ denotes the different polarizations of partons (quark or antiquark) and $U$ stands for kaon being unpolarized. By taking certain limits, Wigner distributions in the impact-parameter plane $\left(\mathbf{b}_{\perp}\right.$ plane) and the transverse momentum plane $\left(\mathbf{k}_{\perp}\right.$ plane) can be explained. In addition, the distributions in the mixed plane, commonly known as mixed probability densities $\rho\left(b_{x}, k_{y}\right)$ or $\rho\left(k_{x}, b_{y}\right)$, can also be explained through

$$
\int d b_{y} d k_{x} \rho_{U X}\left(\mathbf{b}_{\perp}, \mathbf{k}_{\perp}\right)=\rho_{U X}\left(b_{x}, k_{y}\right),
$$

or

$$
\int d b_{x} d k_{y} \rho_{U X}\left(\mathbf{b}_{\perp}, \mathbf{k}_{\perp}\right)=\rho_{U X}\left(k_{x}, b_{y}\right) .
$$

The variables surviving in the mixed distribution, which actually show the correlation between the transverse momentum and transverse coordinate of the quark (antiquark), are not protected by the uncertainty principle. Therefore, they are known to describe the probabilistic distributions.

In Figs. 6-8, we present, respectively the results of Wigner distributions of the unpolarized, longitudinally polarized, and transversely polarized $u$ quark as well as the $\bar{s}$ quark in the unpolarized kaon. In our numerical calculations, the active quark (antiquark) is considered to be the $u$ quark ( $\bar{s}$ quark), and the spectator is the $\bar{s}$ quark ( $u$ quark). Corresponding to Eqs. (40)-(42) for $u$ and Eqs. (44)-(46) for $\bar{s}$, we plot the results in the impactparameter plane, momentum plane, and mixed plane. In the impact-parameter plane, we show the plots of $\rho_{U U}$ [Figs. 6(a) and 6(b)], $\rho_{U L}$ [Figs. 7(a) and 7(b)], and $\rho_{U T}$ [Figs. 8(a) and 8(b)] by taking the fixed transverse momentum as $\quad \mathbf{k}_{\perp}=k_{\perp} \hat{e}_{y} \quad$ where $\quad k_{\perp}=0.2 \mathrm{GeV}$.
Similarly, in the transverse momentum plane we plot $\rho_{U U}$ [Figs. 6(c) and 6(d)], $\rho_{U L}$ [Figs. 7(c) and 7(d)], and $\rho_{U T}$ [Figs. 8(c) and 8(d)] by choosing the impact-parameter coordinate along $\hat{e}_{y}$, i.e., $\mathbf{b}_{\perp}=b_{\perp} \hat{e}_{y}$ and $b_{\perp}=0.4 \mathrm{GeV}^{-1}$. Further, for the mixed plane we plot $\rho_{U U}$ [Figs. 6(e) and 6(f)], $\rho_{U L}$ [Figs. 7(e) and 7(f)], and $\rho_{U T}$ [Figs. 8(e) and 8(f)].

From Fig. 6, where the unpolarized Wigner distribution in the impact-parameter plane, momentum plane, and mixed plane have been plotted for the $u$ quark on the left panel and for the $\bar{s}$ quark on the right panel, we observe that in the case of $\bar{s}$ the distribution is more concentrated at the center in contrast to the $u$ quark. The distributions in both cases have opposite behavior. From Figs. 6(a) and 6(b), the distribution in the impact-parameter plane for the case of $\bar{s}$ does not remain circularly symmetric at the higher values of the transverse coordinates of the $\mathbf{b}_{\perp}$ plane, whereas in case of the $u$ quark, it shows symmetry. This is due to the effect of the heavier mass of the active $\bar{s}$ quark. We observe that the probability of the rotation of the quark (antiquark) to move in either the clockwise direction or the anticlockwise direction is the same. Further, in Figs. 6(c) and 6(d), the distributions $\rho_{U U}$ are presented in the momentum plane. The distributions in this case are observed to be circularly symmetric for both $u$ and $\bar{s}$, but the concentrations are in the opposite direction. In the case of distributions in the mixed plane $\rho\left(b_{x}, k_{y}\right)$, the $u$ and $\bar{s}$ quark distributions are axially symmetric. The spread is, however, more dispersed in the case of the $u$ quark as compared to the $\bar{s}$ quark. At the center, i.e., at $b_{x}=k_{y}=0$, the probability density for the $u$ quark is maximum while in the case of the $\bar{s}$ quark it is minimum. The distribution $\rho_{U U}$ is related to the unpolarized TMD $f_{1}$ and unpolarized GPD $H$, when integrated upon certain limits.

The results of Wigner distributions for the longitudinally polarized quark (antiquark) in the unpolarized kaon viz. $\rho_{U L}$ are shown in Fig. 7. In the impact-parameter plane, the distributions show the dipole behavior and the behavior is positive for $b_{x}>0$ for both quark and antiquark, as shown in Fig. 7(a) and 7(b). However, in the transverse momentum plane, shown in Figs. 7(c) and 7(d), it reverses the direction and $\rho_{U L}$ is positive for $b_{x}<0$. In the mixed plane $\left(b_{x}, k_{y}\right)$, we observe a quadrupole behavior of the distributions in both cases with the same polarities as shown in Figs. 7(e) and 7(f). A positive distribution is observed in the region where the product of $b_{x}$ and $k_{y}$ is greater, whereas it is 


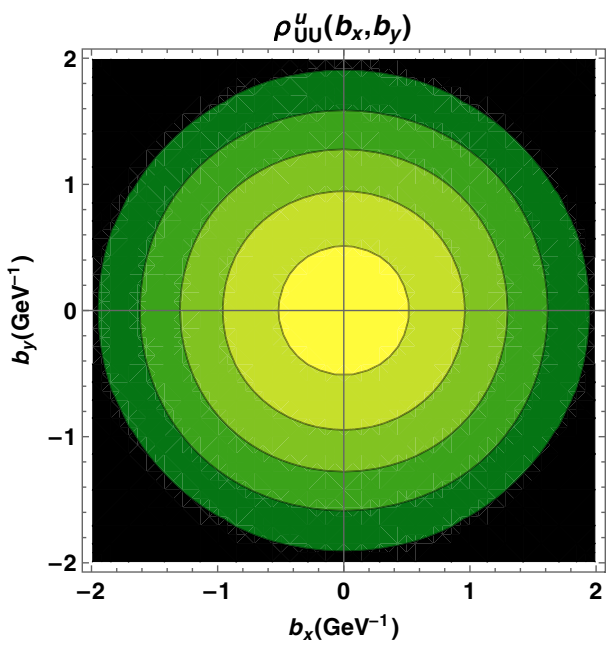

(a)

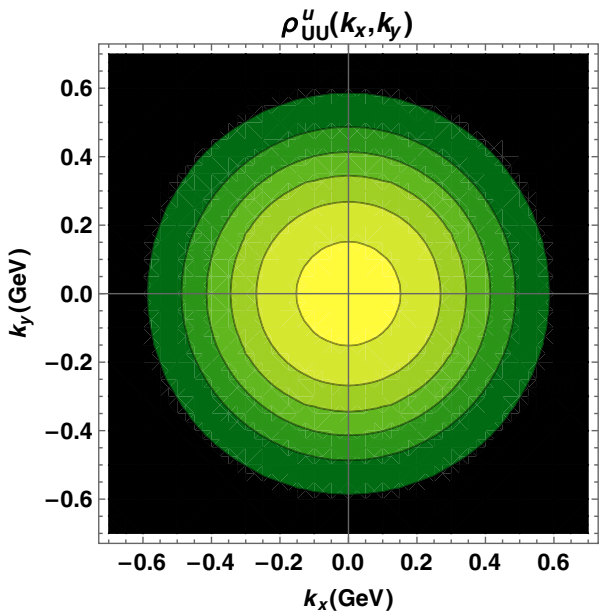

(c)

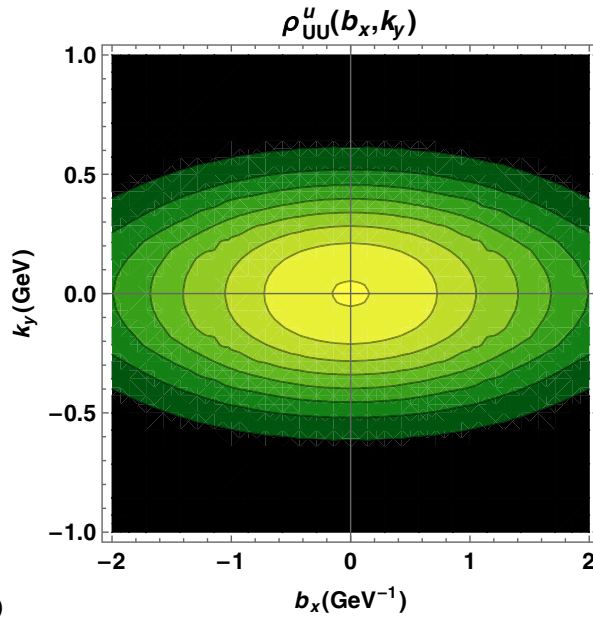

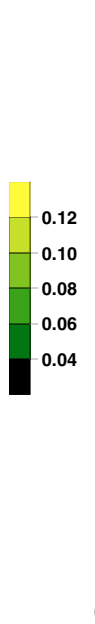

(b)
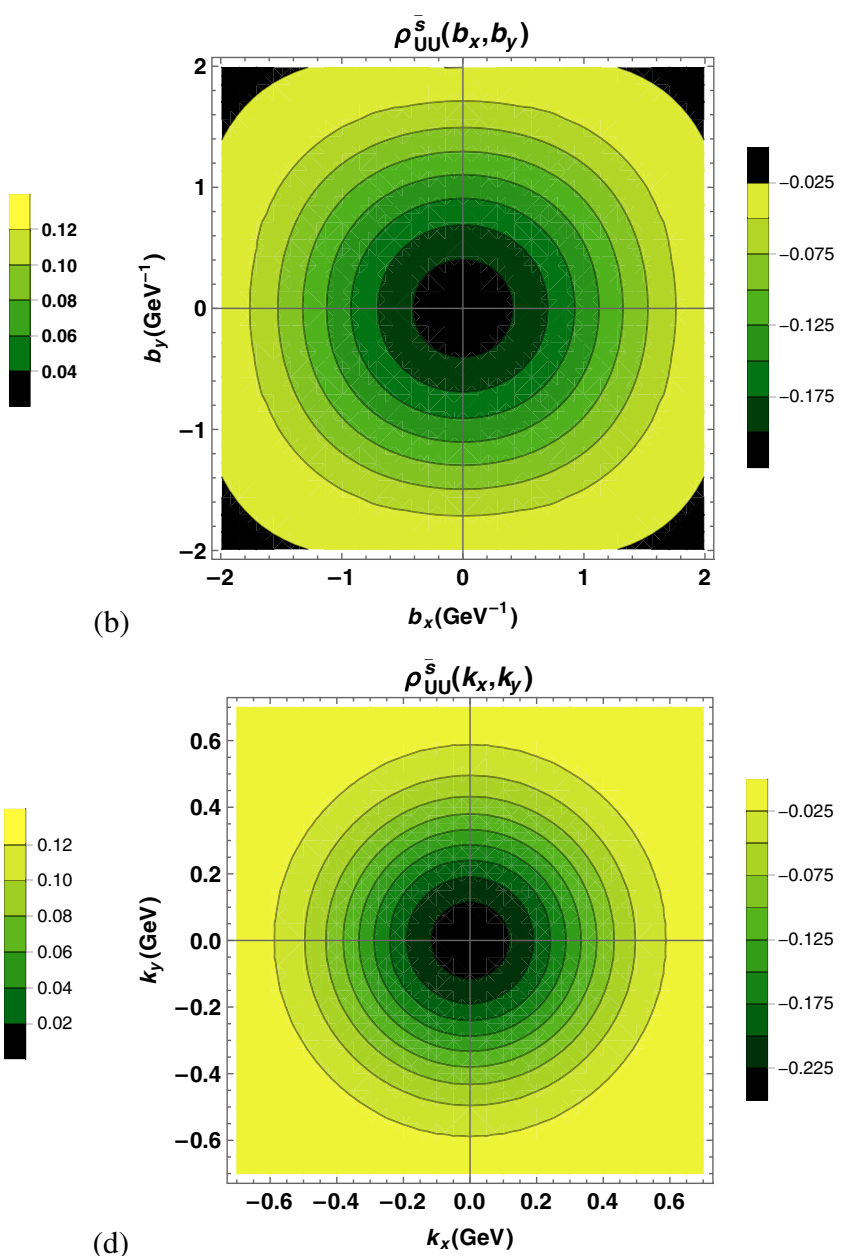

(d)

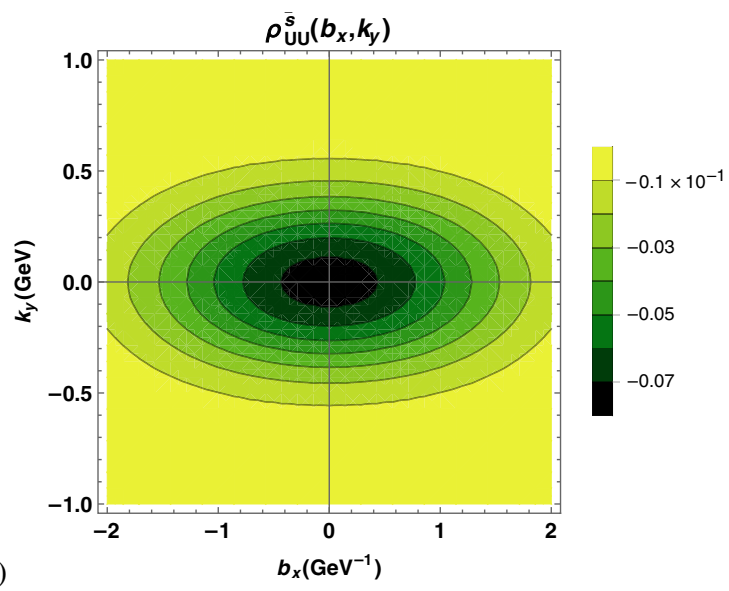

FIG. 6. The unpolarized Wigner distribution $\rho_{U U}$ of the $u$ quark (left panel) and the $\bar{s}$ quark (right panel) for the kaon in the impactparameter plane (a) and (b), the transverse momentum plane (c) and (d), and the mixed plane (e) and (f).

negative where the product is negative. For the probability density we have

$$
\rho_{U L}\left(b_{x}, k_{y}\right)\left\{\begin{array}{ll}
>0 & \text { if } b_{x} * k_{y}>0 \\
<0 & \text { if } b_{x} * k_{y}<0
\end{array} .\right.
$$

No TMD or GPD is present corresponding to $\rho_{U L}$. Generally, this Wigner distribution is related to OAM, but for the pseudoscalar meson, the net quark and antiquark spin and OAM are zero.

We plot the unpolarized-transverse distribution $\rho_{U T}^{j}$ of $u$ and $\bar{s}$, respectively, on the left and right panels in Fig. 8 . 


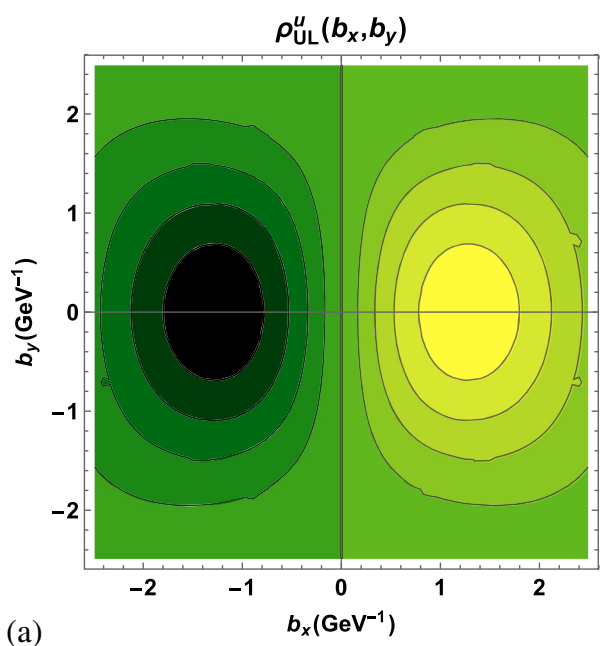

(a)
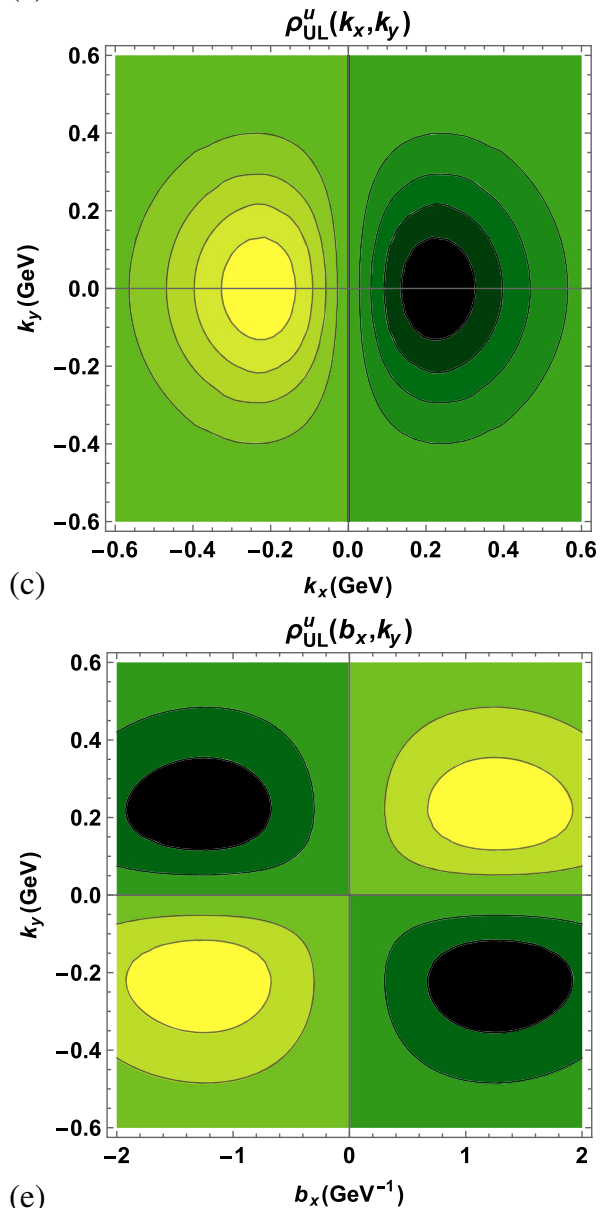
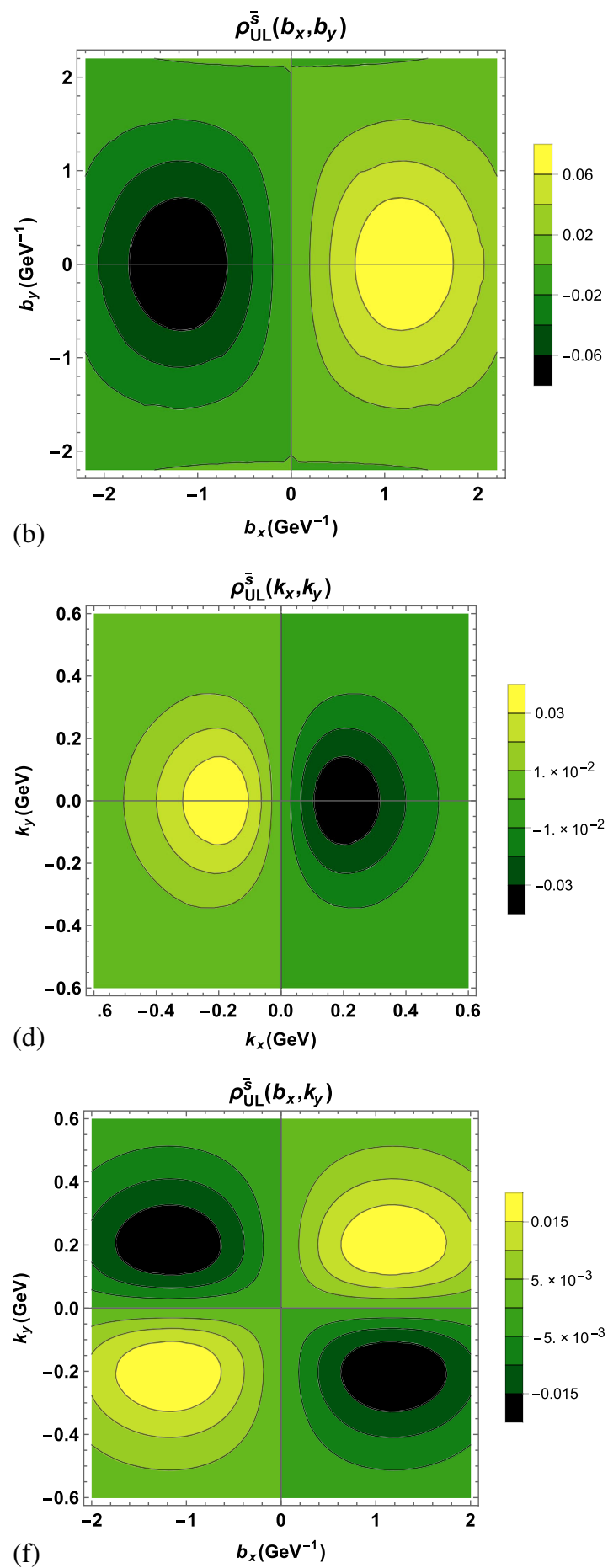

FIG. 7. The unpolarized-longitudinal Wigner distribution $\rho_{U L}$ of the u quark (left panel) and the $\bar{s}$ quark (right panel) for the kaon in the impact-parameter plane (a) and (b), the transverse momentum plane (c) and (d), and the mixed plane (e) and (f).

The distribution $\rho_{U T}^{j}$ sheds light on the quark/antiquark distribution when the quark is transversely polarized in an unpolarized kaon. Here $j$ describes the polarization direction of the quark (antiquark). We take the quark/ antiquark polarization along the $x$ axis. We notice a strong correlation between the transverse coordinate and the perpendicular polarization direction of the quark/antiquark.
From Eqs. (42) and (46), we observe that the distribution vanishes if we consider the quark/antiquark spin direction along the direction of the quark/antiquark transverse coordinate. The distribution displayed in the impactparameter plane shows a dipolar behavior for both the quark and antiquark but with opposite polarities. This is clear from Figs. 8(a) and 8(b). Further, in the transverse 

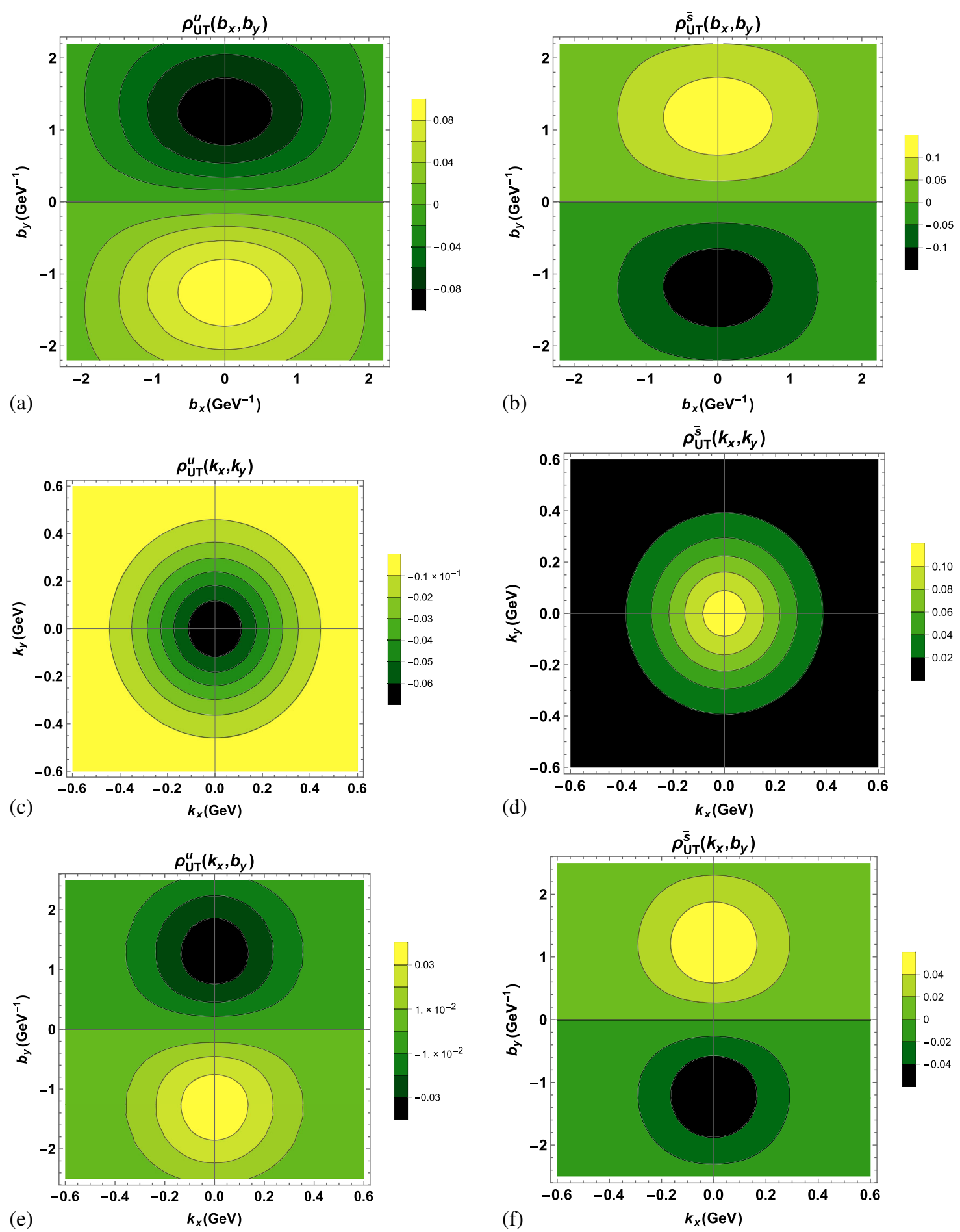

FIG. 8. The unpolarized-transverse Wigner distribution $\rho_{U Y}^{j}$ of the u quark (left panel) and the $\bar{s}$ quark (right panel) for the kaon in the impact-parameter plane (a) and (b), the transverse momentum plane (c) and (d), and the mixed plane (e) and (f).

momentum plane [Figs. 8(c) and 8(d)], $\rho_{U T}^{1}$ is observed to be more focused at the center $\left(p_{x}=p_{y}=0\right)$ in the case of $\bar{s}$ whereas it is more extended to the periphery in the case of the $u$ quark distribution. The distribution shows a dipolar behavior in mixed space due to its symmetry in the momentum plane as well as in the impact-parameter plane.
Here, we choose the plane $\left(k_{x}, b_{y}\right)$ instead of $\left(b_{x}, k_{y}\right)$, because it leads to the Dirac Delta function $\delta\left(\Delta_{y}\right)$ in the case of $\rho_{U T}^{j}$, when the Fourier transformation is taken. $\rho_{U T}^{j}$ relates to the T-odd Boer-Mulder TMD $h_{1}^{\perp}$ and the T-odd GPD $E_{T}$ at the TMD limit and the impact-parameter dependent distribution limit, respectively. But we are not 
able to extract any TMD or GPD in the present work, because we have not considered any gluon contributions.

\section{GENERALIZED TRANSVERSE MOMENTUM- DEPENDENT DISTRIBUTIONS OF $u$ AND $\bar{s}$ QUARKS IN THE KAON}

The twist-2 GTMDs related to the unpolarized pseudoscalar meson with spin- 0 are connected with the Wigner correlator or operator as [33]

$$
\begin{aligned}
\hat{W}^{\left[\gamma^{+}\right]} & =F_{1}, \\
\hat{W}^{\left[\gamma^{+} \gamma_{5}\right]} & =\frac{i \epsilon_{\perp}^{i j} k_{\perp}^{i} \Delta_{\perp}^{j}}{M^{2}} \tilde{G}_{1}, \\
\hat{W}^{\left[i \sigma^{j+} \gamma_{5}\right]} & =\frac{i \epsilon_{\perp}^{i j} k_{\perp}^{i}}{M} H_{1}^{k}+\frac{i \epsilon_{\perp}^{i j} \Delta_{\perp}^{i}}{M} H_{1}^{\Delta},
\end{aligned}
$$

with the antisymmetric tensor $\epsilon_{\perp}^{i j}=\epsilon^{-+i j}, \epsilon^{0123}=1$ and $\sigma^{a b}=\frac{i}{2}\left[\gamma^{a}, \gamma^{b}\right]$. All the leading-twist GTMDs are functions of six variables and we have $\left(x, \zeta, \mathbf{k}_{\perp}^{2}, \mathbf{k}_{\perp} . \boldsymbol{\Delta}_{\perp}, \Delta_{\perp}^{2}\right)$. There are four complex-valued twist-2 GTMDs in the case of the spin-0 hadron, whereas in case of the spin- $\frac{1}{2}$ there are 16.

The explicit expressions of $u$ quark GTMDs for $\zeta \neq 0$ in the kaon are evaluated from

$$
\begin{aligned}
F_{1}^{(u)}= & \frac{1}{16 \pi^{3}}\left[\mathbf{k}_{\perp}^{2}-\left(1-x^{\prime}\right)\left(1-x^{\prime \prime}\right) \frac{\Delta_{\perp}^{2}}{4}\right. \\
& \left.+\frac{x^{\prime \prime}-x^{\prime}}{2}\left(k_{x} \Delta_{x}+k_{y} \Delta_{y}\right)+\mathcal{M}_{u}^{\prime} \mathcal{M}_{u}^{\prime \prime}\right] \\
& \times \frac{\varphi_{u}^{\dagger}\left(x^{\prime \prime}, \mathbf{k}_{\perp}^{\prime \prime}\right) \varphi_{u}\left(x^{\prime}, \mathbf{k}_{\perp}^{\prime}\right)}{\sqrt{\mathbf{k}_{\perp^{\prime \prime}}^{2}+l_{u}^{\prime \prime 2}} \sqrt{\mathbf{k}_{\perp^{\prime}}^{2}+l_{u}^{\prime 2}}} \\
\tilde{G}_{1}^{(u)}= & -\frac{M^{2}}{16 \pi^{3}} \frac{\left(2-x^{\prime}-x^{\prime \prime}\right)}{2} \\
& \times \frac{\varphi_{u}^{\dagger}\left(x^{\prime \prime}, \mathbf{k}_{\perp}^{\prime \prime}\right) \varphi_{u}\left(x^{\prime}, \mathbf{k}_{\perp}^{\prime}\right)}{\sqrt{\mathbf{k}_{\perp^{\prime \prime}}^{2}+l_{u}^{\prime \prime 2}} \sqrt{\mathbf{k}_{\perp^{\prime}}^{2}+l_{u}^{\prime 2}}} \\
H_{1}^{k(u)}= & -\frac{M}{16 \pi^{3}}\left[\mathcal{M}_{u}^{\prime}-\mathcal{M}_{u}^{\prime \prime}\right] \frac{\varphi_{u}^{\dagger}\left(x^{\prime \prime}, \mathbf{k}_{\perp}^{\prime \prime}\right) \varphi_{u}\left(x^{\prime}, \mathbf{k}_{\perp}^{\prime}\right)}{\sqrt{\mathbf{k}_{\perp}^{2}}+l_{u}^{\prime \prime 2}} \sqrt{\mathbf{k}_{\perp^{\prime}}^{2}+l_{u}^{\prime 2}} \\
H_{1}^{\Delta(u)}= & \frac{M}{16 \pi^{3}}\left[\mathcal{M}_{u}^{\prime} \frac{\left(1-x^{\prime \prime}\right)}{2}+\mathcal{M}_{u}^{\prime \prime} \frac{\left(1-x^{\prime}\right)}{2}\right] \\
& \times \frac{\varphi_{u}^{\dagger}\left(x^{\prime \prime}, \mathbf{k}_{\perp}^{\prime \prime}\right) \varphi_{u}\left(x^{\prime}, \mathbf{k}_{\perp}^{\prime}\right)}{\sqrt{\mathbf{k}_{\perp^{\prime \prime}}^{2}+l_{u}^{\prime \prime 2}} \sqrt{\mathbf{k}_{\perp^{\prime}}^{2}+l_{u}^{\prime 2}}} .
\end{aligned}
$$

The $\bar{s}$ quark GTMDs in the kaon are related to the $u$ quark distributions as Eqs. (20) and (43). We have

$$
\begin{aligned}
& F^{u}\left(x, \zeta, \mathbf{k}_{\perp}^{2}, \mathbf{k}_{\perp} . \boldsymbol{\Delta}_{\perp}, \boldsymbol{\Delta}_{\perp}^{2}, m_{1}, m_{2}\right) \\
& \quad=-F^{\bar{s}}\left(-x, \zeta, \mathbf{k}_{\perp}^{2},-\mathbf{k}_{\perp} . \boldsymbol{\Delta}_{\perp}, \boldsymbol{\Delta}_{\perp}^{2}, m_{2}, m_{1}\right) .
\end{aligned}
$$

The $\bar{s}$ quark twist-2 GTMDs for $\zeta \neq 0$ are explicitly evaluated from

$$
\begin{aligned}
F_{1}^{(\bar{s})}= & -\frac{1}{16 \pi^{3}}\left[\mathbf{k}_{\perp}^{2}-\left(1-x^{\prime}\right)\left(1-x^{\prime \prime}\right) \frac{\Delta_{\perp}^{2}}{4}\right. \\
& \left.+\frac{x^{\prime \prime}-x^{\prime}}{2}\left(k_{x} \Delta_{x}+k_{y} \Delta_{y}\right)+\mathcal{M}_{\bar{s}}^{\prime} \mathcal{M}_{\bar{s}}^{\prime \prime}\right] \\
& \times \frac{\varphi_{\bar{s}}^{\dagger}\left(x^{\prime \prime}, \mathbf{k}_{\perp}^{\prime \prime}\right) \varphi_{\bar{s}}\left(x^{\prime}, \mathbf{k}_{\perp}^{\prime}\right)}{\sqrt{\mathbf{k}_{\perp \prime}^{2}+l_{\bar{s}}^{\prime \prime 2}} \sqrt{\mathbf{k}_{\perp^{\prime}}^{2}+l_{\bar{s}}^{\prime 2}}}, \\
\tilde{G}_{1}^{(\bar{s})}= & \frac{M^{2}}{16 \pi^{3}} \frac{\left(2-x^{\prime}-x^{\prime \prime}\right)}{2} \frac{\varphi_{\bar{s}}^{\dagger}\left(x^{\prime \prime}, \mathbf{k}_{\perp}^{\prime \prime}\right) \varphi_{\bar{s}}\left(x^{\prime}, \mathbf{k}_{\perp}^{\prime}\right)}{\sqrt{\mathbf{k}_{\perp^{\prime \prime}}^{2}+l_{\bar{s}}^{\prime \prime 2}} \sqrt{\mathbf{k}_{\perp^{\prime}}^{2}+l_{\bar{s}}^{\prime 2}}}, \\
H_{1}^{k(\bar{s})}= & \frac{M}{16 \pi^{3}}\left[\mathcal{M}_{\bar{s}}^{\prime}-\mathcal{M}_{\bar{s}}^{\prime \prime}\right] \frac{\varphi_{\bar{s}}^{\dagger}\left(x^{\prime \prime}, \mathbf{k}_{\perp}^{\prime \prime}\right) \varphi_{\bar{s}}\left(x^{\prime}, \mathbf{k}_{\perp}^{\prime}\right)}{\sqrt{\mathbf{k}_{\perp^{\prime \prime}}^{2}+l_{\bar{s}}^{\prime \prime 2}} \sqrt{\mathbf{k}_{\perp^{\prime}}^{2}+l_{\bar{s}}^{\prime 2}}} \\
H_{1}^{\Delta(\bar{s})}= & -\frac{M}{16 \pi^{3}}\left[\mathcal{M}_{\bar{s}}^{\prime} \frac{\left(1-x^{\prime \prime}\right)}{2}+\mathcal{M}_{\bar{s}}^{\prime \prime} \frac{\left(1-x^{\prime}\right)}{2}\right] \\
& \times \frac{\varphi_{\bar{s}}^{\dagger}\left(x^{\prime \prime}, \mathbf{k}_{\perp}^{\prime \prime}\right) \varphi_{\bar{s}}\left(x^{\prime}, \mathbf{k}_{\perp}^{\prime}\right)}{\sqrt{\mathbf{k}_{\perp^{\prime \prime}}^{2}+l_{\bar{s}}^{\prime \prime 2}} \sqrt{\mathbf{k}_{\perp^{\prime}}^{2}+l_{\bar{s}}^{\prime 2}}} .
\end{aligned}
$$

The details of the longitudinal momentum fractions and transverse momenta carried by the quark (antiquark) have already been given in Sec. III in Eqs. (14)-(19).

In the present work, we have presented the quark and antiquark GTMDs of the kaon with respect to the longitudinal momentum fraction carried by quark $x$ and antiquark $-x$. We have studied two cases: (a) the variation of GTMDs with respect to $x$ for $\zeta \neq 0$ where $\zeta=-\frac{\Delta^{+}}{2 P^{+}}$is the parameter corresponding to the transfer of the momentum to the kaon in the longitudinal direction and (b) the variation of GTMDs with respect to $x$ for $\zeta=0$. It is well known that the so-called mother distributions, i.e., GTMDs, are reducible to the GPDs and TMDs after suitable integrations. For the case when $\zeta \neq 0$, only the GPDs can be extracted since there is no parameter corresponding to the longitudinal direction in the case of TMDs (TMDs do not include $\zeta$ contribution).

In Fig. 9, we have presented the variation of GTMDs of the $u$ and $\bar{s}$ quarks in the kaon corresponding to Eqs. (54)-(57) and Eqs. (59)-(62), respectively. Since the distributions have the support interval $-1<x<1$, we restrict ourselves in the DGLAP regions $(-1<x<-\zeta)$ for the antiquark and for the $(\zeta<x<1)$ quark. In the left 


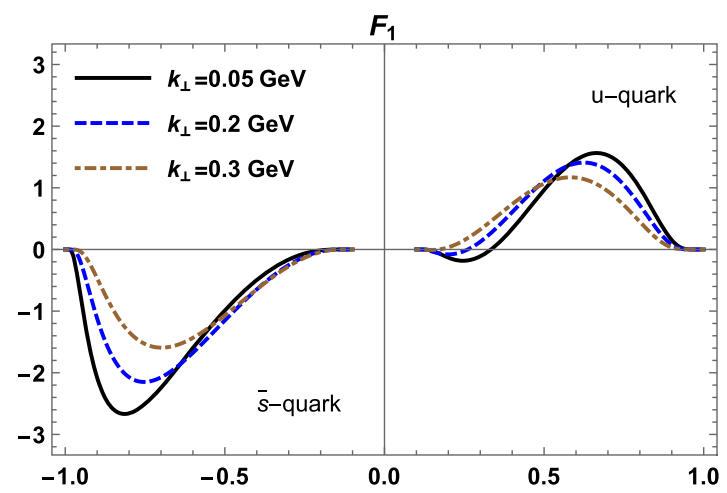

(a)

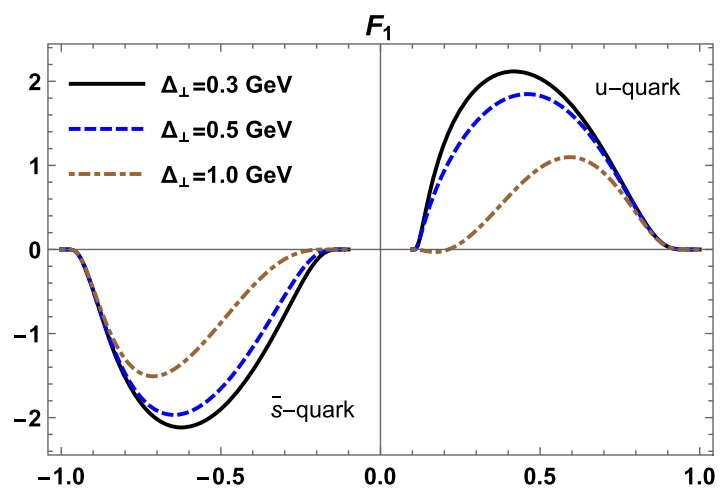

(b)

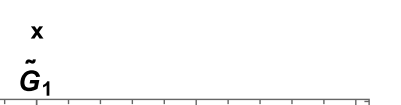

$\mathbf{x}$
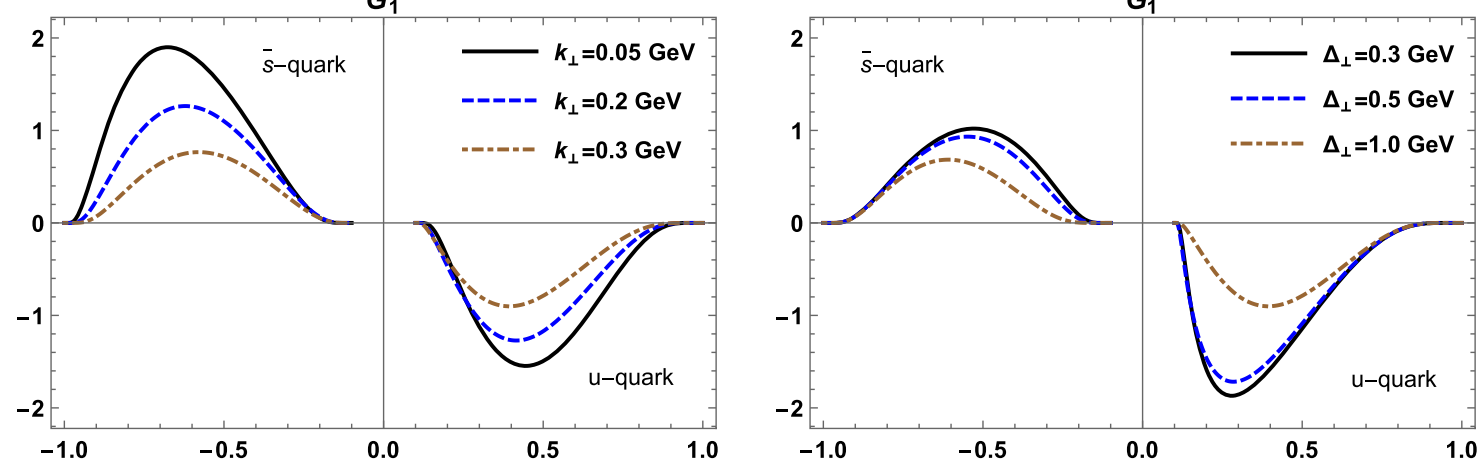

(c) $\quad \mathbf{x}$

(d)
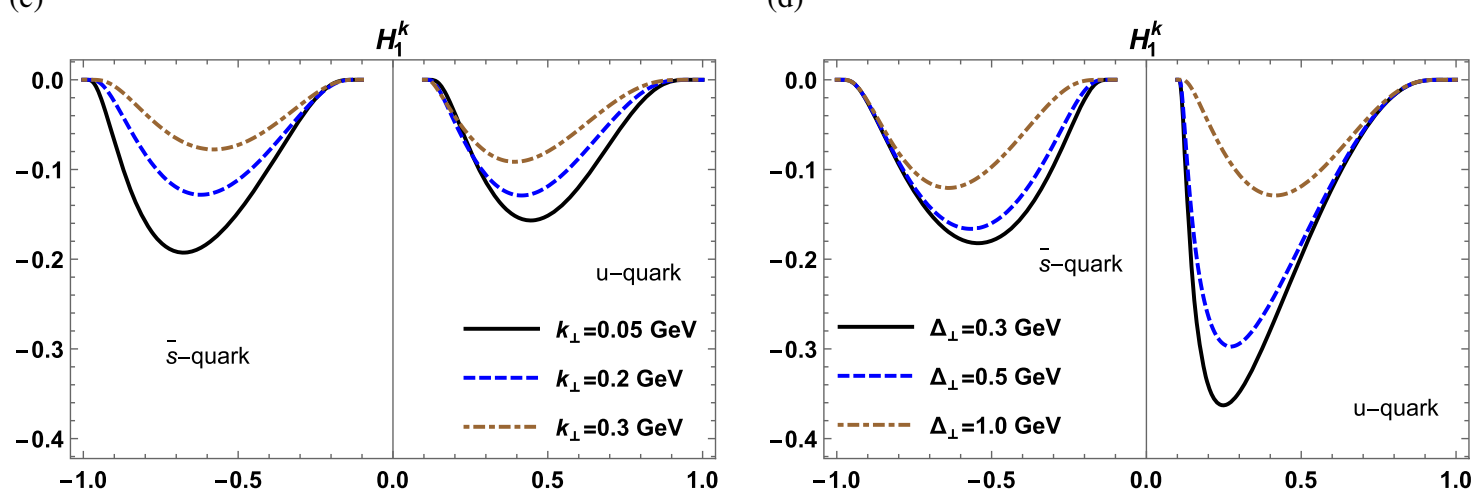

(e)

(f)

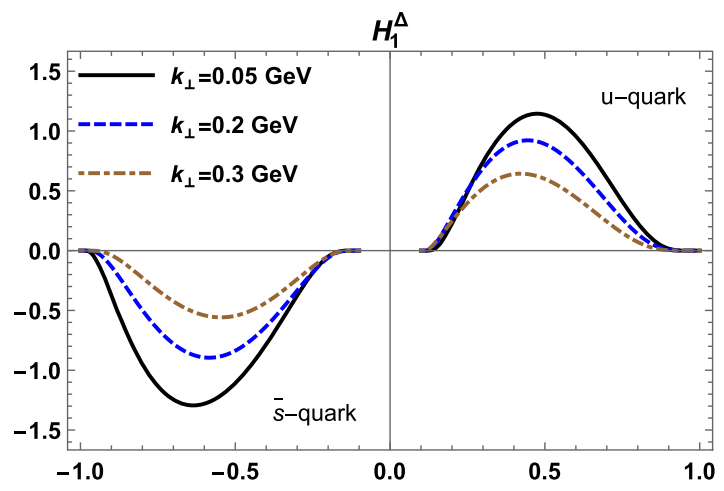

(g)

$\mathbf{x}$

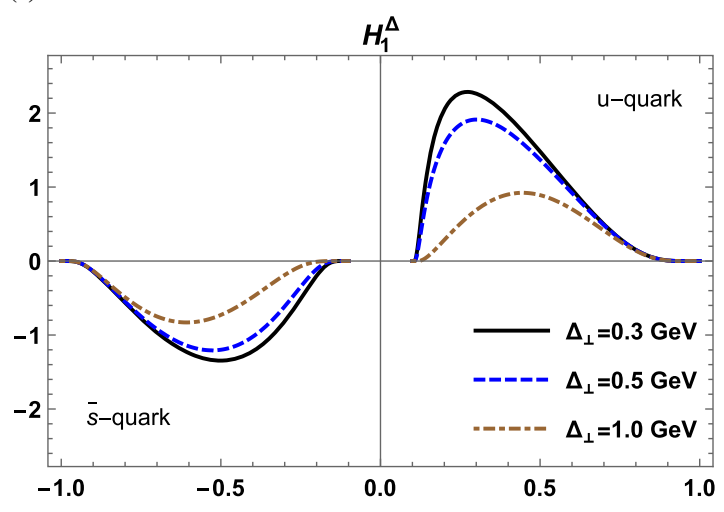

(h)

FIG. 9. The plots of GTMDs $F_{1}\left(x, \zeta, \mathbf{k}_{\perp}^{2}, \mathbf{k}_{\perp} . \boldsymbol{\Delta}_{\perp}, \boldsymbol{\Delta}_{\perp}^{2}\right), \tilde{G}_{1}\left(x, \zeta, \mathbf{k}_{\perp}^{2}, \mathbf{k}_{\perp} . \boldsymbol{\Delta}_{\perp}, \Delta_{\perp}^{2}\right), H_{1}^{k}\left(x, \zeta, \mathbf{k}_{\perp}^{2}, \mathbf{k}_{\perp} . \boldsymbol{\Delta}_{\perp}, \Delta_{\perp}^{2}\right)$, and $H_{1}^{\Delta}\left(x, \zeta, \mathbf{k}_{\perp}^{2}, \mathbf{k}_{\perp}\right.$. $\left.\boldsymbol{\Delta}_{\perp}, \boldsymbol{\Delta}_{\perp}^{2}\right)$ for $\zeta=0.1$ with respect to $x$ for $u$ and $\bar{s}$ quarks at different values of $\mathbf{k}_{\perp}$ with fixed $\boldsymbol{\Delta}_{\perp}=1 \mathrm{GeV}((\mathrm{a}),(\mathrm{c})$, (e), and (g)) as well as at different values of $\boldsymbol{\Delta}_{\perp}$ with fixed $\mathbf{k}_{\perp}=0.2 \mathrm{GeV}$ ((b), (d), (f), and (h)). 
panel of Fig. 9, we have plotted the $u$ quark and $\bar{s}$ quark distributions $F_{1}, \tilde{G}_{1}, H_{1}^{k}$, and $H_{1}^{\Delta}$ for fixed values of $\zeta=0.1$ and $\boldsymbol{\Delta}_{\perp}=1 \mathrm{GeV}$ and at different values of $\mathbf{k}_{\perp}$ at $\mathbf{k}_{\perp}=0.05,0.2$ and $0.3 \mathrm{GeV}$. In all the distributions, we observe that for both quark and antiquark the distribution peaks shift toward the lower values of $x$ and the magnitude also lowers down with the increasing of the quark transverse momentum. For the cases of $F_{1}, \tilde{G}_{1}$, and $H_{1}^{\Delta}$, the effect of the $\bar{s}$ quark is in the opposite direction as compared to the $u$ quark [Figs. 9(a), 9(c), and 9(g)]. The exception lies in the case of $H_{1}^{k}$ [Figs. 9(e) and 9(f)] where the distributions remain negative for both the quark and antiquark in the kaon. In all the cases, at $x=\zeta$, when the momentum fraction carried by the quark (antiquark) is equal to the momentum transferred to the kaon in the longitudinal direction, the distributions vanish. As the longitudinal momentum transfer to the kaon increases, so will the shift in the peaks of the distributions with increasing quark (antiquark) transverse momentum. On the right panel of Fig. 9 [Figs. 9(b), 9(d), 9(f), and 9(h)], the quark (antiquark) GTMDs in the kaon for $\zeta \neq 0$ are displayed at a constant value of $\mathbf{k}_{\perp}=0.2 \mathrm{GeV}$ and by varying $\boldsymbol{\Delta}_{\perp}$. Unlike the distribution, the behavior of the $u$ and $\bar{s}$ quarks, by varying $\mathbf{k}_{\perp}$ and keeping $\boldsymbol{\Delta}_{\perp}$ constant, the distribution peaks move

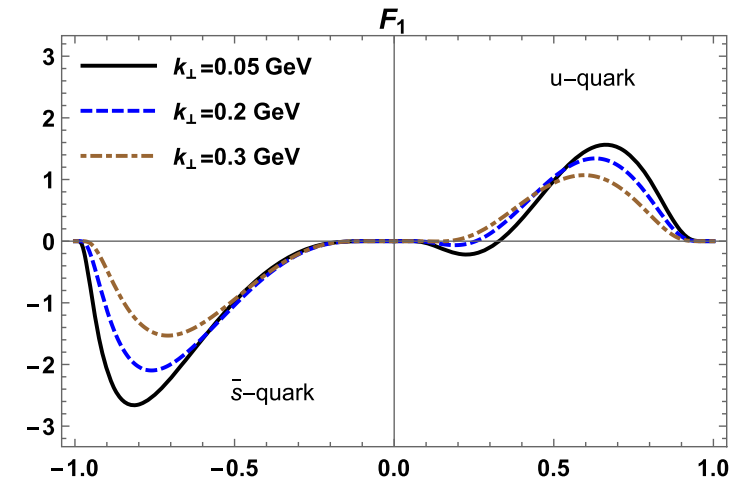

(a)

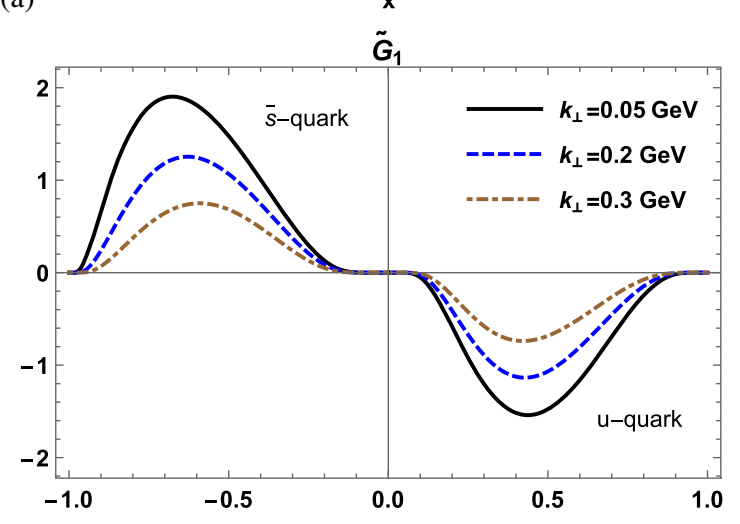

(c)

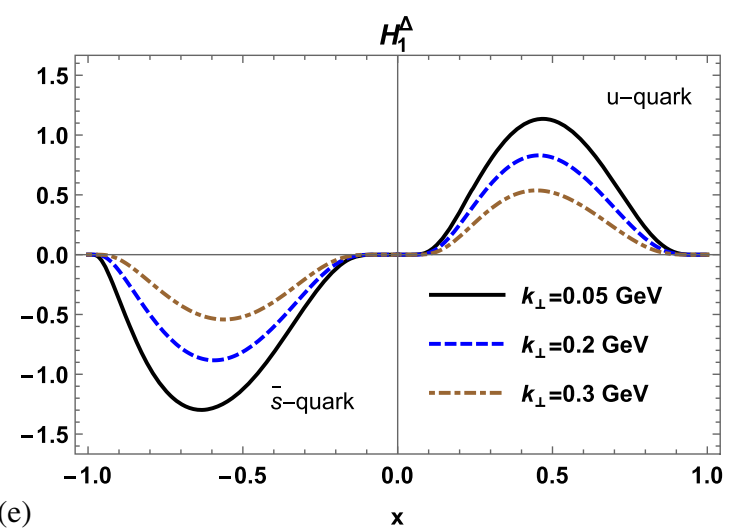

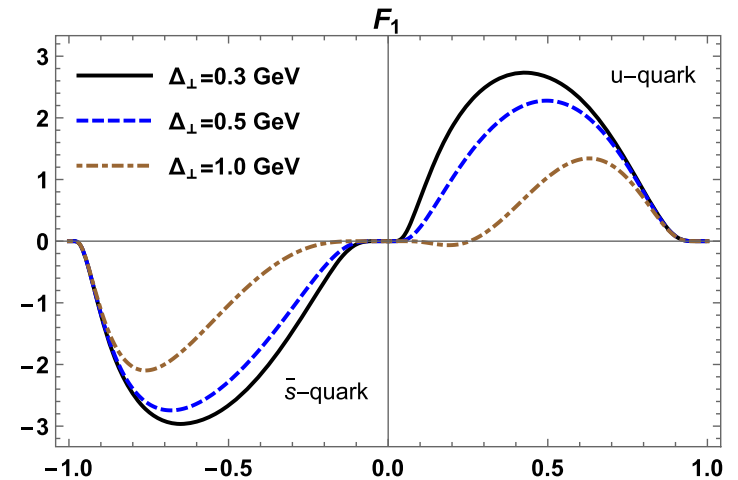

(b)

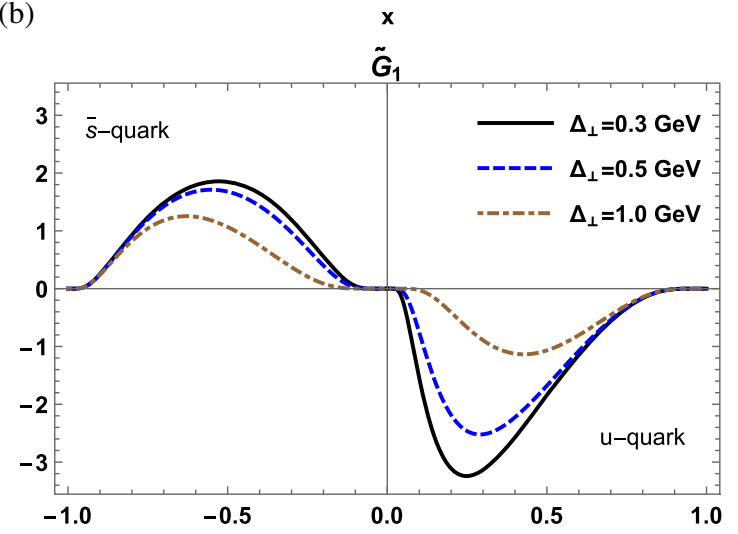

(d)

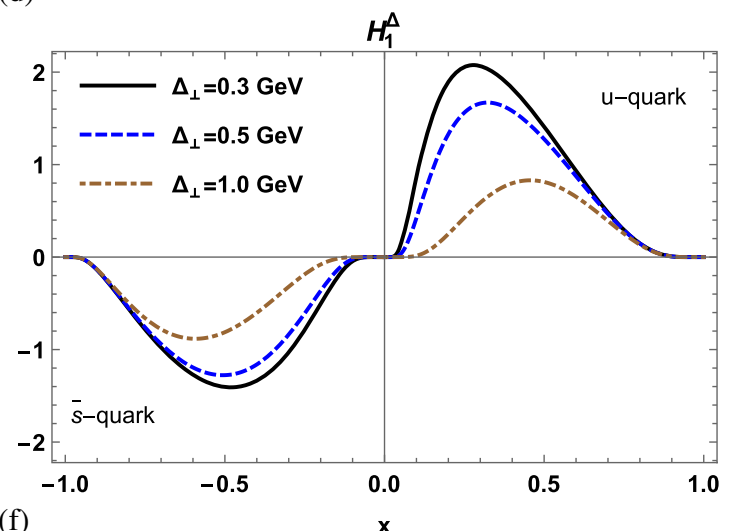

(f)

FIG. 10. The plots of GTMDs $F_{1}\left(x, 0, \mathbf{k}_{\perp}^{2}, \mathbf{k}_{\perp} . \boldsymbol{\Delta}_{\perp}, \Delta_{\perp}^{2}\right), \tilde{G}_{1}\left(x, 0, \mathbf{k}_{\perp}^{2}, \mathbf{k}_{\perp} \cdot \boldsymbol{\Delta}_{\perp}, \Delta_{\perp}^{2}\right)$, and $H_{1}^{\Delta}\left(x, 0, \mathbf{k}_{\perp}^{2}, \mathbf{k}_{\perp} . \boldsymbol{\Delta}_{\perp}, \Delta_{\perp}^{2}\right)$ with respect to $x$ for $u$ and $\bar{s}$ quarks at different values of $\mathbf{k}_{\perp}$ with fixed $\boldsymbol{\Delta}_{\perp}=1 \mathrm{GeV}\left((\mathrm{a})\right.$, (c), and (e)) as well as at different values of $\boldsymbol{\Delta}_{\perp}$ with fixed $\mathbf{k}_{\perp}=0.2 \mathrm{GeV}((\mathrm{b}),(\mathrm{d})$, and (f)). 
toward the higher values of $x$ when the total momentum transferred to the kaon is increased. The magnitude of the distribution, however, decreases when the total momentum transfer to the final state of kaon is increased. Here, the magnitude of the $\bar{s}$ quark is less as compared to the $u$ quark. The difference in the quark and antiquark distributions shifts for $\zeta \neq 0$ is due to the heavy on-shell mass of the active antiquark as well as the momentum transfer to the kaon in the longitudinal direction leading to the dependence of the mass of the active quark on the distributions when the total momentum transfer to the kaon is fixed.

In Fig. 10, we show the graphical presentation of GTMDs $F_{1}, \tilde{G}_{1}, H_{1}^{\Delta}$ for $\zeta=0$. For $\zeta=0$, the complexvalued twist-2 GTMDs reduce to 3 in number as is evident from Eqs. (56) and (61). The distribution peaks show the same behavior for both $u$ and $\bar{s}$ quarks when $\mathbf{k}_{\perp}$ is varied and $\boldsymbol{\Delta}_{\perp}$ is kept constant except for the magnitudes and polarities. This implies that there are no on-shell mass effects when the longitudinal momentum transfer is absent.
On the other hand, when the momentum transfer to the kaon is varied by keeping $\mathbf{k}_{\perp}$ constant, the effect on the distributions corresponding to $x$ remains same as for $\zeta \neq 0$. It is known that the GTMDs corresponding to $\zeta=0$ are the Fourier transformations of Wigner distributions. Therefore, the Wigner distribution $\rho_{U U}$ corresponds to the unpolarized GTMD $F_{1}$, from which one can evaluate the other unpolarized probabilistic distributions. As $\tilde{G}_{1}$ relates to $\rho_{U L}$, it would lead to the spin and orbital angular momentum correlation, the detailed description of which has been discussed in the next section.

\section{SPIN-ORBIT CORRELATION}

Following the study of the spin-orbit correlation of the quark in a proton $[55,59,71,72]$ and pion [61], we study the spin-orbit correlation of the $u$ and $\bar{s}$ quarks in the kaon. The correlation between the quark spin and quark OAM is defined in terms of an operator as [55,59]

$$
C_{z}^{q}\left(b^{-}, \mathbf{b}_{\perp}, k^{+}, \mathbf{k}_{\perp}\right)=\frac{1}{2} \int \frac{d z^{-} d^{2} \mathbf{z}_{\perp}}{(2 \pi)^{3}} e^{i k \cdot z} \bar{\psi}^{q}\left(b^{-}-\frac{z^{-}}{2}, \mathbf{b}_{\perp}\right) \gamma^{+} \gamma^{5}\left(\mathbf{b}_{\perp} \times\left(-i \stackrel{\leftrightarrow}{\partial}{ }_{\perp}\right)\right) \psi^{q}\left(b^{-}+\frac{z^{-}}{2}, \mathbf{b}_{\perp}\right)
$$

The quark spin-orbit correlation in terms of Wigner distributions can be written as

$$
C_{z}^{q}=\int d x d^{2} \mathbf{k}_{\perp} d^{2} \mathbf{b}_{\perp}\left(\mathbf{b}_{\perp} \times \mathbf{k}_{\perp}\right)_{z} \rho^{\left[\gamma^{+} \gamma^{5}\right]}\left(\mathbf{b}_{\perp}, \mathbf{k}_{\perp}, x\right) .
$$

Following Eq. (35), we can write the above equation in terms of the Wigner distribution of a longitudinally polarized quark in an unpolarized kaon as

$$
C_{z}^{q}=\int d x d^{2} \mathbf{k}_{\perp} d^{2} \mathbf{b}_{\perp}\left(\mathbf{b}_{\perp} \times \mathbf{k}_{\perp}\right)_{z} \rho_{U L}\left(\mathbf{b}_{\perp}, \mathbf{k}_{\perp}, x\right) .
$$

The unpolarized-longitudinal Wigner distribution is parametrized in terms of the GTMD $\tilde{G}_{1}$ as

$$
\rho_{U L}\left(\mathbf{b}_{\perp}, \mathbf{k}_{\perp}, x\right)=\frac{1}{M^{2}} \epsilon_{\perp}^{i j} k_{\perp}^{i} \frac{\partial}{\partial b_{\perp}^{j}} \tilde{\mathcal{G}}_{1}\left(x, 0, \mathbf{k}_{\perp}^{2}, \mathbf{k}_{\perp} \cdot \mathbf{b}_{\perp}, \mathbf{b}_{\perp}^{2}\right),
$$

where

$$
\begin{aligned}
& \tilde{\mathcal{G}}_{1}\left(x, 0, \mathbf{k}_{\perp}^{2}, \mathbf{k}_{\perp} \cdot \mathbf{b}_{\perp}, \mathbf{b}_{\perp}^{2}\right) \\
& \quad=\int \frac{d^{2} \boldsymbol{\Delta}_{\perp}}{(2 \pi)^{2}} e^{-i \boldsymbol{\Delta}_{\perp} \cdot \mathbf{b}_{\perp}} \tilde{G}_{1}\left(x, 0, \mathbf{k}_{\perp}^{2}, \mathbf{k}_{\perp} . \boldsymbol{\Delta}_{\perp}, \Delta_{\perp}^{2}\right) .
\end{aligned}
$$

In terms of GTMDs, the definition of the quark spin-orbit correlator is given as

$$
C_{z}^{q}=\int d x d^{2} \mathbf{k}_{\perp} \frac{\mathbf{k}_{\perp}^{2}}{M^{2}} \tilde{G}_{1}\left(x, 0, \mathbf{k}_{\perp}, 0,0\right) .
$$

The case $C_{z}>0$ favors the alignment of the quark spin and OAM. Otherwise, for $C_{z}<0$, it leads to the antialignment of the quark spin and OAM.

In Fig. 11, we have plotted the correlation $C_{z}$ with respect to the longitudinal momentum fraction $x$. Here we study how the correlation between the spin and OAM varies with $x$. There seems to be a strong correlation between the $\bar{s}$ quark spin and OAM near the central values of the longitudinal momentum fraction carried by the $\bar{s}$ quark, i.e., $-x$. However, for the $u$ quark, the correlation peak is negative and is observed at lower values of the quark

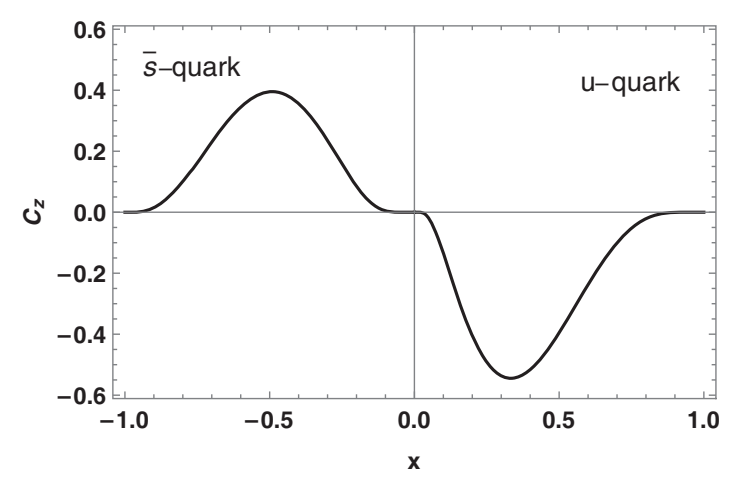

FIG. 11. The spin-orbit correlation $\left(C_{z}\right)$ of the $u$ quark and $\bar{s}$ quark in the kaon with respect to $x$. 
longitudinal momentum fraction, i.e., $x$. When $C_{z}$ is integrated upon $x$, the value comes out to be $C_{z}^{\bar{s}}=0.176$ and $C_{z}^{u}=-0.234$, which implies that the $\bar{s}$ quark OAM is parallel to the $\bar{s}$ quark spin and $u$ quark OAM is antiparallel to the $u$ quark spin. If we compare these results with the previous studies for the case of the proton, it is found that the spin-orbit correlations for $u$ and $d$ quarks are negative and antialigned in both the cases in the light-front quarkdiquark model inspired from AdS/QCD [55,59]. In the light-cone constituent quark model and light-cone chiral quark-soliton model, $C_{z}$ is positive for both quarks in the case of the proton [71]. Recently, the spin-OAM correlation has been discussed for the pion in the light-cone quark model (in Ref. [61]), where they have $C_{z}^{q}=-0.159$ with the quark spin being antiparallel to the quark OAM. Our results are in line with these observations.

\section{SUMMARY AND CONCLUSIONS}

In this paper, we have studied the various dimensional distributions of $u$ and $\bar{s}$ quarks in the kaon using the lightcone quark model. We evaluate three-dimensional probabilistic generalized parton distributions for the valence $u$ and $\bar{s}$ quarks by considering the presence of the longitudinal momentum transferred to the kaon's final state $(\zeta \neq 0)$. Also, the quasiprobabilistic distributions, the Wigner distributions and the mother distributions GTMDs, are also discussed for the case of $u$ and $\bar{s}$ quarks in the kaon. The momentum-space wave function used in the present work is associated with the Brodsky-HuangLepage prescription. The parameters used in the present work are able to give experimentally consistent results of electromagnetic form factors. As the support interval for the distributions is $-1<x<1$, we chose the DGLAP regions, $-1<x<-\zeta$ and $\zeta<x<1$, respectively, for the antiquark and quark. Here, $x$ defines the longitudinal momentum fraction carried by the parton. For the calculations of the $u$ quark distribution, the $\bar{s}$ quark is considered as a spectator, and vice versa.

For the case of the unpolarized GPD of quark and antiquark for nonzero skewedness, we study the distribution as a function of the variable $x$ by considering different values of $-t$ in one case and with different values of $\zeta$ in the other case. A shift in the distribution peak is observed along higher magnitudes of $x$ when there is an increase in the total momentum transfer to the kaon. The height of the peak, however, decreases. We have also presented the variation of GPD as a function of $x$ but at a fixed $-t=0.5$ and different values of $\zeta$. We observe a high peak at the lower value $\zeta$. Therefore, if the momentum transfer along the longitudinal direction is less, the spread is found to be maximum. Further, we discussed the quark and antiquark distributions at $x=0.7$ and $x=-0.7$ as a function of $\zeta$ for different values of $-t$. It is observed that at $\zeta=0$ the distribution is different for different total momentum transferred to the final state of the kaon. In all the cases, we observe that the $\bar{s}$ quark amplitudes are comparatively large and in the opposite direction to the case of $u$ quark.

The relation of the impact-parameter dependent GPDs are derived by taking the Fourier transformation of unpolarized GPD and they have been studied as a function of $x$ and transverse distance from the center of the kaon, i.e., $\mathbf{b}_{\perp}$. We observe the absence of the distribution of valence quarks when the transverse distance from the center of the kaon is large. On the other hand, it is maximum when the transverse distance is small. Another important observation for the distribution of $u$ and $\bar{s}$ quarks is that the spread moves toward the lower $x$ for higher values of $\mathbf{b}_{\perp}$. The distribution peak of the $\bar{s}$ quark is localized at higher values of $x$ as compared to the $u$ quark which is because of the heavier active quark mass in the case of the bars quark. Further, the 3D distribution for the transverse distance distribution as a function of $\zeta$ and $\mathbf{b}_{\perp}$ at a constant value of the longitudinal momentum fraction $x$ (for quark) and $-x$ (for antiquark) is maximum when no momentum is transferred to the final state of the kaon for both quarks. For $\zeta=0$, the impact-parameter dependent GPD converts to give the parton distribution $q\left(x, \mathbf{b}_{\perp}\right)$. The spread is increased when the antiquark longitudinal momentum is higher toward negative polarities and in the middle of the transverse impact parameter. While for the quark, the spread is near the center with respect to the quark longitudinal momentum fraction.

Further, the transverse Wigner distributions $\rho\left(\mathbf{b}_{\perp}, \mathbf{k}_{\perp}\right)$ for the unpolarized kaon, with the unpolarized, longitudinally polarized, and transversely polarized composites, i.e., the $u$ quark and $\bar{s}$ quark, have been presented graphically in the transverse impact-parameter plane, transverse momentum plane, and mixed plane. For $\rho_{U U}$, the distribution comes out to be circularly symmetric about the center in the $\mathbf{b}_{\perp}$ and $\mathbf{k}_{\perp}$ planes. However, in the mixed plane, the axially symmetric distribution is observed. The $\rho_{U L}$ displays a dipolar distribution in the $\mathbf{b}$ plane as well as in the $\mathbf{k}_{\perp}$ plane. The quadrupole distribution is observed in the mixed plane which is related to the spin-orbital angular momentum correlation of partons in the kaon. The polarization direction was taken along the $x$ axis to evaluate $\rho_{U T}^{j}$, i.e., $j=1$. A dipolar distribution was observed in the transverse impact-parameter plane and the mixed plane whereas a circularly symmetric distribution in the transverse momentum plane focused at the $k_{x}=k_{y}=0$. The spread is more concentrated in the case of $\bar{s}$ which is because of the heavy mass of the strange quark in comparison with the up quark. The polarities seem to be opposite for both as the relation consumes the negative sign for strange quark distributions. The exception lies in case of $\rho_{U L}$. The strong correlation between the partons and kaon are well indicated by the phase-space distributions in the context of the transverse momentum and transverse impact-parameter coordinates. The probabilistic distributions are possible to extract from the Wigner distributions upon certain limits. 
Furthermore, GTMDs related to the Fourier transformation of Wigner distributions for $\zeta=0$ and entitled as mother distributions, help in extracting the corresponding GPDs and TMDs by applying some limits. We have investigated the relation of unpolarized kaon GTMDs with $x$ at different values of $\mathbf{k}_{\perp}$ and $\boldsymbol{\Delta}_{\perp}$ for $u$ and $\bar{s}$ quarks. There are four GTMDs for the case of the kaon: $F_{1}$, $\tilde{G}_{1}, H_{1}^{k}$, and $H_{1}^{\Delta}$. In all the observations, we get to know that by increasing the quark (antiquark) transverse momentum $\mathbf{k}_{\perp}$, the distribution peaks move backward toward the lower values of $x$ with a decrease in their magnitudes. While observing the distributions corresponding to $x$ at different $\boldsymbol{\Delta}_{\perp}$, the trend becomes opposite. By increasing the momentum transfer $\boldsymbol{\Delta}_{\perp}$, the distribution peaks move forward toward the higher values of $x$ while the magnitudes decrease. The $u$ quark and $\bar{s}$ quark distributions have opposite polarities except for the case of $H_{1}^{k}$. The distribution $H_{1}^{k}$ become zero for $\zeta=0$. GTMD $F_{1}$ is related to the Wigner distribution $\rho_{U U}$ through the Fourier transformation and further, at certain limits, GPD $H$ and TMD $f_{1}$ come into picture. There is no GPD or TMD corresponding to $\rho_{U L}$ and $\tilde{G}_{1}$ but they are instead connected to the spin-orbit correlation $C_{z}$. The values come out to be $C_{z}=0.176$ for the $\bar{s}$ quark and $C_{z}=-0.234$ for the $u$ quark. From this it can be concluded that the $\bar{s}$ quark's spin and OAM are antialigned whereas the $u$ quark's spin and OAM are aligned to each other. Also no GPD and TMD related to $\rho_{U T}^{j}$ and $H_{1}^{k}$ and $H_{1}^{\Delta}$ are present in this model which is because of the absence of any gluon interaction.

To conclude, the results presented in the manuscript can perhaps be substantiated further by future measurements for the kaon where the quark GTMDs can be extracted through the exclusive double Drell-Yan process where the final state must carry the two virtual photons. The GPDs can be accessible through deeply virtual Compton scattering and DVMP processes.

\section{ACKNOWLEDGMENTS}

S. K. would like to thank M. Diehl for helpful discussions. H. D. would like to thank the Department of Science and Technology (Reference No. EMR/2017/001549) Government of India for financial support.
[1] J. C. Collins and D. E. Soper, Nucl. Phys. B194, 445 (1982).

[2] A. D. Martin, W. J. Stirling, R. S. Thorne, and G. Watt, Eur. Phys. J. C 63, 189 (2009).

[3] C. F. Perdrisat, V. Punjabi, and M. Vanderhaeghen, Prog. Part. Nucl. Phys. 59, 694 (2007).

[4] M. Diehl and P. Kroll, Eur. Phys. J. C 73, 2397 (2013).

[5] M. Diehl, T. Feldmann, R. Jakob, and P. Kroll, Eur. Phys. J. C 39, 1 (2005).

[6] M. Guidal, M. V. Polyakov, A. V. Radyushkin, and M. Vanderhaeghen, Phys. Rev. D 72, 054013 (2005).

[7] A. Radyushkin, Phys. Rev. D 56, 5524 (1997).

[8] X. Ji, Phys. Rev. Lett. 78, 610 (1997).

[9] M. Diehl, Phys. Rep. 388, 41 (2003).

[10] A. V. Belitsky and A. V. Radyushkin, Phys. Rep. 418, 1 (2005).

[11] B. Sigfrido and B. Pasquini, Riv. Nuovo Cimento 30, 387 (2007).

[12] J. Collins, Int. J. Mod. Phys. Conf. Ser. 04, 85 (2011).

[13] S. Meissner, A. Metz, and K. Geoke, Phys. Rev. D 76, 034002 (2007).

[14] M. Diehl, Eur. Phys. J. C 25, 223 (2002); 31, 277(E) (2003).

[15] M. Burkardt, Phys. Rev. D 62, 071503 (2000).

[16] J. Blümlein, Prog. Part. Nucl. Phys. 69, 28 (2013).

[17] X. Ji, Phys. Rev. D 55, 7114 (1997).

[18] M. Mazouz, A. Camsonne, C. Muõz Camacho et al. (Jefferson Lab Hall A Collaboration), Phys. Rev. Lett. 99, 242501 (2007).

[19] M. Defurne et al., Nat. Commun. 8, 1408 (2017).
[20] K. Kumericki, S. Liuti, and H. Moutarde, Eur. Phys. J. A 52, 157 (2016).

[21] R. Dupre, M. Guidal, and M. Vanderhaeghen, Phys. Rev. D 95, 011501 (2017).

[22] P. Kroll, EPJ Web Conf. 85, 01005 (2015).

[23] C. Hadjidakis (CLAS Collaboration), Phys. Lett. B 605, 256 (2005).

[24] S. Chekanov (ZEUS Collaboration), Nucl. Phys. B718, 3 (2005).

[25] A. Airapetian (HERMES Collaboration), Phys. Lett. B 659, 486 (2008).

[26] A. Bacchetta, Eur. Phys. J. A 52, 163 (2016).

[27] R. Ent, Eur. Phys. J. A 52, 162 (2016).

[28] R. A. Martinez et al., Acta Phys. Pol. B 46, 2501 (2015).

[29] M. Boglione and A. Prokudin, Eur. Phys. J. A 52, 154 (2016).

[30] C. A. Aidala, S. D. Bass, D. Hasch, and G. K. Mallot, Rev. Mod. Phys. 85, 655 (2013).

[31] X. Ji, Phys. Rev. Lett. 91, 062001 (2003).

[32] A. V. Belitsky, X. Ji, and F. Yuan, Phys. Rev. D 69, 074014 (2004).

[33] S. Meissner, A. Metz, M. Schlegel, and K. Goeke, J. High Energy Phys. 08 (2008) 038.

[34] S. Meissner, A. Metz, and M. Schlegel, J. High Energy Phys. 08 (2009) 056.

[35] S. Bhattacharya, A. Metz, and Jian Zhou, Phys. Lett. B 771, 396 (2017).

[36] X. Ji, W. Melnitchouk, and X. Song, Phys. Rev. D 56, 5511 (1997). 
[37] S. Scopetta and V. Vento, Eur. Phys. J. A 16, 527 (2003).

[38] S. Scopetta and V. Vento, Phys. Rev. D 69, 094004 (2004).

[39] S. Boffi, B. Pasquini, and M. Traini, Nucl. Phys. B649, 243 (2003).

[40] S. Boffi, B. Pasquini, and M. Traini, Nucl. Phys. B680, 147 (2004).

[41] B. Pasquini and S. Boffi, Phys. Rev. D 73, 094001 (2006).

[42] C. Mondal and D. Chakrabarti, Eur. Phys. J. C 75, 261 (2015).

[43] T. Gutsche, V. E. Lyubovitskij, I. Schmidt, and A. Vega, J. Phys. G 42, 095005 (2015).

[44] N. Kaur, N. Kumar, C. Mondal, and H. Dahiya, Nucl. Phys. B934, 80 (2018).

[45] L. Theussl, S. Noguera, and V. Vento, Eur. Phys. J. A 20 , 483 (2004).

[46] B. C. Tiburzi and G. A. Miller, Phys. Rev. D 67, 054014 (2003).

[47] W. Broniowski and E. R. Arriola, Phys. Lett. B 574, 57 (2003).

[48] A. E. Dorokhov, W. Broniowski, and E. R. Arriola, Phys. Rev. D 84, 074015 (2011).

[49] N. Kumar and H. Dahiya, Phys. Rev. D 91, 114031 (2015).

[50] N. Kumar and H. Dahiya, Int. J. Mod. Phys. A 30, 1550010 (2015).

[51] H. Dahiya, A. Mukherjee, and S. Ray, Phys. Rev. D 76, 034010 (2007).

[52] C. Lorcé, B. Pasquini, and M. Vanderhaeghen, J. High Energy Phys. 05 (2011) 041.

[53] C. Lorcé, B. Pasquini, X, Xiong, and F. Yuan, Phys. Rev. D 85, 114006 (2012).
[54] T. Liu and B.-Q. Ma, Phys. Rev. D 91, 034019 (2015).

[55] D. Chakrabarti, T. Maji, C. Mondal, and A. Mukherjee, Phys. Rev. D 95, 074028 (2017).

[56] J. More, A. Mukherjee, and S. Nair, Phys. Rev. D 95, 074039 (2017).

[57] N. Kumar and C. Mondal, Nucl. Phys. B931, 226 (2018).

[58] S. Kaur and H. Dahiya, Nucl. Phys. B937, 272 (2018).

[59] D. Chakrabarti, T. Maji, C. Mondal, and A. Mukherjee, Eur. Phys. J. C 76, 409 (2016).

[60] S. Kaur and H. Dahiya, arXiv:1906.04662.

[61] Z.-L. Ma and Z. Lu, Phys. Rev. D 98, 054024 (2018).

[62] P. T.P. Hutaurak, Non-Perturbative Aspects of Kaon Structure, Ph.D. Thesis, The University of Adelaide, 2016.

[63] J. T. Londergan, G. Q. Liu, and A. W. Thomas, Phys. Lett. B 380, 393 (1996).

[64] P. A. M. Dirac, Rev. Mod. Phys. 21, 392 (1949).

[65] W.-M. Zhang, Chin. J. Phys. (Taipei) 32, 717 (1994).

[66] S. J. Brodsky, D. S. Hwang, B.-Q. Ma, and I. Schmidt, Nucl. Phys. B593, 311 (2001).

[67] B.-W. Xiao, X. Qian, and B.-Q. Ma, Eur. Phys. J. A 15, 523 (2002).

[68] W. Qian and B.-Q. Ma, Phys. Rev. D 78, 074002 (2008).

[69] S. J. Brodsky, M. Diehl, and D. S. Hwang, Nucl. Phys. B596, 99 (2001).

[70] S. J. Brodsky and G. F. de Teramond, Phys. Rev. D 77, 056007 (2008).

[71] C. Lorcé and B. Pasquini, Phys. Rev. D 84, 014015 (2011).

[72] C. Lorcé, Int. J. Mod. Phys. Conf. Ser. 37, 1560036 (2015). 